\title{
Multi-Period Stock Allocation Via Robust Optimization
}

\author{
Peter L. Jackson and John A. Muckstadt
}

March, 2015

\begin{abstract}
In this paper we re-visit a long-standing multi-echelon inventory allocation problem from a robust optimization perspective. We formulate the problem as a one warehouse, $N$-retailer, multi-period, stock allocation problem in which holding costs are identical at each location and no stock is received from outside suppliers for the duration of the planning horizon. Stock may be transferred from the central warehouse to the retailers instantaneously and without cost at the beginning of each period for which the central warehouse still has stock on hand. No other stock transfers are allowed. Under this set-up, the only motive for holding inventory at the central warehouse for allocation in future periods is the so-called risk-pooling motive. The dynamic programming formulation of this problem requires a state space too large for practical computation. Various approximation methods have been proposed for variants of this problem. We apply robust optimization to this problem extending the typical uncertainty set to capture the risk pooling phenomenon and extending the inventory policy to allow for an adaptive, non-anticipatory shipment policy. We show how to represent the uncertainty set compactly so that it grows by no more than the square of the number of retailers. The problem can be solved using Benders' decomposition in the general case. In the special case of no initial retailer inventories, two periods, and identical retailers, a relaxed form of the problem admits a closed form solution with surprising insights. Summarizing the experimental results of the paper, we see both confirmation of the value of the robust optimization approach as well as managerial insights into the design and operation of multi-echelon inventory systems.
\end{abstract}

\section{Introduction}

During the past half-century, researchers have studied multi-echelon inventory allocation problems extensively. Surveys of this literature can be found in [Axsäter, 2003] and [Doğru et al., 2009]. For periodic review systems, Clark and Scarf, in their seminal paper [Clark and Scarf, 1960], developed a decomposition approach that, under certain conditions, provides a mechanism for analyzing and 
solving a two-echelon central warehouse and multiple retailer distribution system problem. The modeling approach they employed provided a way to find an optimal procurement and allocation policy for the two echelon system under the so-called balance assumption. Basically, balance occurs when there is no desire to move, or re-allocate, stock from one retailer to another in any time period. Thus, if $x_{i t}$ is the echelon stock at retailer $i$ at the beginning of period $t$ and $y_{i t}$ is the desired order-up-to level for that retailer in period $t$, then balance occurs if $y_{i t} \geq x_{i t}$ for all retailers. Suppose the central warehouse does not place orders with its supplier every period. The time between placement of these upper echelon orders is called a cycle. [Jönsson and Silver, 1987] made the observation that for items having relatively low coefficients of variation in their daily demand processes, inventory imbalance will occur among retailers, if at all, only at the end of a cycle. This has led to many formulations of the problem with only two periods: a lengthy first period during which imbalance at the retailer locations can be ignored followed by a short period during which imbalance may be an issue at these locations. If imbalance is an issue, then it is necessary to include constraints requiring shipments to retailers to be non-negative. The inclusion of these constraints leads to a dynamic programming problem formulation that is computationally intractable for realistic problem sizes. The balance assumption, on the other hand, permits the problem to be decomposed into single-location problems. Numerous papers have exploited the balance assumption, beginning with [Clark and Scarf, 1960], [Eppen and Schrage, 1981], [Federgruen and Zipkin, 1984a], and [Federgruen and Zipkin, 1984b]. An analytical investigation of the imbalance assumption, including the impact of demand correlation, can be found in [Zipkin, 1984]. A more recent review and application of the balance assumption can be found in [Gallego et al., 2007].

[Doğru et al., 2009] survey the papers in this area in order to highlight the widespread use of the balance assumption. To test the applicability of the balance assumption they compute a gap between an upper and lower bound on the optimal expected cost. The lower bound is based on the relaxation approach of Federguen and Zipkin and relies on the balance assumption. The upper bound is determined by simulating a policy based on the optimal solution to the relaxed problem but which myopically optimizes allocations subject to the non-negative shipment constraints. The authors find that the gap is small for identical retailers if the coefficient of variation of demand is small or moderate (less than 0.5) or if the incremental holding cost at the retailers is high. The gap is small for non-identical retailers only in a few of their parameters settings, even with moderate coefficients of variation. This suggests that either the lower bound or the heuristic upper bound policy, or both, are mediocre in settings characterized by high coefficients of variation or non-identical retailers.

A number of authors have attempted to incorporate the non-negative shipment constraints to improve the optimization results. [Jackson and Muckstadt, 1989] restrict attention to a two-period allocation cycle and show that when nonnegative shipments are enforced, the maximum post-allocation stockout probability across retailers converges to a constant as the number of retailers increases. Using this constant, they develop an approximate cost function which is sepa- 
rable by retailer and can be optimized to set their target inventory levels.

[Axsäter et al., 2002] introduce a two-step allocation heuristic which is also based on a two-period allocation cycle. Target allocation levels for the first period, $y_{i}(u)$, for each retailer $i$ are determined for different values of $u$, the total allocation in the first period, to optimize cost myopically in the first period. For each vector of realized demands in period 1, the optimal second period allocations can be determined as a function of these target allocation levels, enforcing the non-negative shipment constraints. Using convolutions of a three-point probability function to represent the joint probability distribution of period 1 demands, the expected cost associated with period 2 can then be approximated. Summing the period 1 and period 2 costs, the total cost can be tabulated as a function of $u$, the initial allocation, and, from this, the optimal value of $u$ can be chosen. The optimally-chosen $u$ is then allocated to retailers in a myopically optimal way. The best length for the first period is determined experimentally.

Using a more general multi-period approach, [Kunnumkal and Topaloglu, 2008] develop a lower bound on the cost of the optimal policy by associating Lagrange multipliers with the non-negative shipment constraints. The resulting dynamic programming problem is separable into single-location dynamic programs which are solved easily. Subgradient optimization is then used to find a vector of Lagrange multipliers which maximizes the lower bound. [Kunnumkal and Topaloglu, 2011] acknowledge the computational difficulty of the subgradient optimization step in this approach and instead use much faster linear programming approaches to find good Lagrange multipliers. The best of these methods requires Monte Carlo simulation of realized demand vectors, solving a linear program for each realized demand vector, extracting the Lagrange multipliers from the dual solution, and averaging these multiplier values.

One important instance of the central warehouse, multi-retailer problem is the following. The central warehouse is replenished once in each cycle of a lengthy time horizon. Each cycle consists of multiple periods. For example, a cycle could be a week in duration and a period could be a day in length. The question then is how much inventory should be allocated to each retailer so as to minimize costs. Suppose that there are two types of costs, holding and backorder costs. Furthermore, suppose the holding cost rate is the same at the central warehouse as it is at each of the retailers. In this case, there is no economic incentive to hold inventory at the central warehouse. The only motive for holding inventory at the central warehouse is to reduce the risk of inventory imbalance. Holding inventory back at the central warehouse is a form of risk pooling. Suppose we make the Clark- Scarf balance assumption. Then, when holding costs are equal among all locations, and the balance assumption holds, it is easy to show that all inventory received at the central warehouse will be immediately allocated to the retailers. This is the policy first employed for this situation by Eppen and Schrage.

Suppose, on the other hand, we do not make the balance assumption. To study this problem for the environment we have stated, we use a robust optimization approach. Robust optimization has been proposed as a tractable 
optimization approach for stochastic planning problems that are too large to be solved by dynamic programming. Proponents of robust optimization make several claims for the approach:

1. Robust optimization models lead to solutions whose expected cost is close to the minimum expected cost in cases where the optimum can be computed, especially when compared to the error possible from selecting probability distribution models incorrectly;

2. Robust optimization models do not suffer the curse of dimensionality which plagues expected cost optimization models; in many cases, the dimension of the robust optimization formulation is no larger than the size of a corresponding deterministic model.

3. Robust optimization models in special cases lead to closed form solutions which provide analytical insight into the underlying problems.

All three of these benefits, for example, are demonstrated in [Bertsimas and Thiele, 2006]. The problem with applying this approach directly to stock allocation problems is that it is a static planning problem: all shipments to all retailers are set at one time. Worst case performance is determined relative to these fixed shipments. As a result, the value of withholding inventory for the purpose of risk-pooling is not captured. What is needed in the representation is an adaptive policy: one in which shipments will depend on the evolution of demand across time and retailers.

In [Bredström et al., 2013], the current status of adaptive policies for robust optimization is reviewed. The authors propose a general approach for modeling planning problems with right hand side uncertainty, specifically, demand uncertainty. In their approach, variables are partitioned into business decisions, to be made without knowledge of actual demand, and recourse decisions, to be made after revelation of the actual demands. The partition results in a master problem which is a linear program and a subproblem which is a bilinear program. They propose using Benders' decomposition approach to solve the problem and rely on iterative techniques to solve the bilinear program subproblems. We use a similar approach in this paper, dividing the decisions between inventory policy variables and actual stock allocation decisions. The former are chosen before demands are realized and the latter are consequences of the policy variables and the actual demands. We too use Benders' decomposition to solve the overall problem and we face a bilinear program for the subproblem. In our case, the simplicity of our formulation allows us to recast the bilinear program as a mixed-integer linear program.

Our primary contribution to modeling inventory systems using robust optimization is to include the phenomenon of risk pooling across retailers. In particular, we develop a computationally tractable approach, via robust optimization, to recommend stock allocations from a central warehouse to multiple retailers over multiple time periods when imbalance is a distinct possibility. Recall that we assume that holding costs are the same at all locations in order to focus solely 
on the risk-pooling motive for holding stock centrally, distinct from economic motivations. We also assume that demand distributions are characterized by their means and variances and that demands are independent across retailers and across time. We find that the claimed benefits of robust optimization, with some qualification, do materialize for this problem. In particular,

1. Stock allocation decisions recommended by this approach capture a large fraction of the risk-pooling benefit that is possible in an expected value sense;

2. The approach is capable of quickly solving problems far beyond the reach of dynamic programming methods; however, to adequately capture the risk-pooling effect, the model size grows with the square of the number of retailers; and

3. A closed form solution is possible for a relaxed version of a two-period, identical-retailer stock allocation problem; this solution provides analytical insight not seen before in the study of this problem.

That is, all the benefits of robust optimization emerge except that the problem size is not proportional to the size of a corresponding deterministic model.

In the course of evaluating the performance of the robust allocation approach, we consider a variety of situations in which risk pooling, and the balance assumption, may or may not play a role in system performance. This study reinforces some conclusions from earlier studies, but also points to new managerial insights. For example, [Doğru et al., 2009] show the balance assumption to be violated to a significant extent when demand among retailers is unbalanced, even for moderate coefficients of variation. Our results point to a different conclusion, and this can be traced to our contention that coefficients of variation are inversely correlated with demand rates, as we have observed in several practical instances.

The remainder of this paper is organized as follows. In section 2, we restrict attention to a single period stock allocation problem in order to review some basic ideas pertaining to expected value-based optimization models. We also introduce a possible robust optimization formulation and contrast the form of its optimal solution with the optimal expected value-based solution. In section 3, we extend the one period models for both approaches, expected value and robust optimization, to handle multi-period problems. The expected value formulation is a dynamic program with a large state space. The robust optimization formulation includes an uncertainty set which captures the risk pooling effect and an adaptive, non-anticipatory shipment policy. The model can be represented as a linear program with a potentially large number of constraints. In Section 4, we propose solution techniques. For the general case, we apply a Benders' decomposition approach to solve the linear form of the general multi-period robust optimization stock allocation problem. The master problem is a simple linear program and the sub-problem is a mixed integer-linear program (MILP). We then consider a special case in which there are no initial inventories at the 
retailers and only two periods in the planning horizon. For the purpose of developing analytical insight, we further simplify this two-period model by assuming the retailers have identical demand distribution parameters in each period, although the parameters may differ between periods. A closed form solution is available for a relaxed version of this problem. The analytical insight is that the worst case from an allocation perspective is that exactly half of the retailers will receive an allocation in the second period and the other half will be in a situation of imbalance. In Section 5, we report on a numerical study which suggests that the recommended stock allocations from the robust optimization approach capture much of the risk-pooling benefit possible, in an expected value sense. Solution times of the robust optimization approach are shown to be quite short although they increase with the number of retailers considered. The numerical studies also give rise to managerial insights into the role of risk pooling in the design of distribution systems. In Section 6, we summarize the results and suggest further research. Many of the results of the paper are established using standard proof approaches and are not instructive. We collect such proofs in an appendix to allow a more focused presentation in the main body of the paper.

\section{Single-Period Stock Allocation}

We begin by considering a single period problem in which a central warehouse allocates stock at the beginning of the period to meet uncertain demand which will occur during the period at each retailer. We introduce some nomenclature and assumptions in the context of an expected value formulation of the problem, state the form of the optimal policy, and then present a robust optimization formulation of the problem. We compare the optimal policies of the two formulations and establish a condition under which the two policies will lead to the same allocation decision.

\subsection{Expected Value Optimization}

Let the set of retailers be denoted by $\mathcal{N}$ and indexed by $i \in \mathcal{N}$. Let $N$ denote the number of retailers: $N=|\mathcal{N}|$, the cardinality of $\mathcal{N}$. Let $v_{i}, i \in \mathcal{N}$, denote the beginning net inventory level (possibly negative) at retailer $i$ and $x_{i}$ denote the allocation of stock from the central warehouse to retailer $i$ at the beginning of the single period. Let $x=\left(x_{i}\right)$, the vector of all stock allocations. Assuming distribution lead times are zero time units in length, the new inventory level at retailer $i$ is thus $v_{i}+x_{i}$ for each retailer, $i \in \mathcal{N}$. A random demand $\widetilde{d}_{i}$ is then observed at each retailer and backorders, if any, are given by $B_{i}$ :

$$
B_{i}=\left(\widetilde{d}_{i}-v_{i}-x_{i}\right)^{+}
$$

where $x^{+}=\max (0, x)$. The warehouse begins the period with a quantity of stock, $v_{0}$, held in reserve. Remember, we require that the allocations to retailers be non-negative and that their total does not exceed the stock held in 
reserve. We assume the cost of holding inventory is the same for an item at all locations in the system and that there are no fixed costs associated with the allocation. Given these assumptions, a reasonable objective is to minimize the sum of expected backorders. Under these conditions, let $f_{1}(v)$ denote the minimal expected sum of retailer backorders at the end of the period when the period begins with inventory vector $v=\left(v_{0}, v_{1}, \ldots, v_{n}\right)$ :

$$
f_{1}(v)=\min _{\substack{x_{i} \geq 0, i \in \mathcal{N} \\ \sum_{i \in \mathcal{N}} x_{i} \leq v_{0}}} \sum_{i \in \mathcal{N}} E\left[\left(\widetilde{d}_{i}-v_{i}-x_{i}\right)^{+}\right] .
$$

Suppose demand at each retailer is represented by a random variable whose probability distribution is expressed in terms of two parameters, its mean and its standard deviation. Specifically, let $\left\{\widetilde{\varepsilon}_{i}, i \in \mathcal{N}\right\}$ be a set of identically distributed, mean-zero, unit-variance, random variables such that the demand at each retailer is given by:

$$
\widetilde{d}_{i}=\mu_{i}+\sigma_{i} \widetilde{\varepsilon}_{i}
$$

for suitable means, $\mu_{i}$, and standard deviations, $\sigma_{i}$, for each $i \in \mathcal{N}$. Let $G(\varepsilon)$ denote the cumulative probability distribution function of $\widetilde{\varepsilon}_{i}$, common to all retailers $i \in \mathcal{N}$; let $\bar{G}(\varepsilon)=1-G(\varepsilon)$ denote its complement, and $\bar{G}^{-1}(\theta)$ denote its inverse. We assume the cumulative probability distribution is continuous and $\bar{G}(\varepsilon)>0$ for all $\varepsilon$.

Proposition 1 Any optimal solution, $x^{*}$, is an equal fractile solution among retailers receiving allocations. That is, there will exist a constant $\theta$ such that

$$
x_{i}^{*}=\left(\mu_{i}+\bar{G}^{-1}(\theta) \sigma_{i}-v_{i}\right)^{+}
$$

for each $i \in \mathcal{N}$. Furthermore, the optimal solution will satisfy

$$
\sum_{i \in \mathcal{N}} x_{i}^{*}=v_{0}
$$

Proof. Appendix 6.2.

Suppose the

$$
y_{i}(\theta)=\mu_{i}+\bar{G}^{-1}(\theta) \sigma_{i}
$$

for each retailer $i \in \mathcal{N}$, for a given value of the parameter $\theta$. We can interpret $y_{i}(\theta)$ as the target stock level for retailer $i$. If $v_{i}<y_{i}(\theta)$ then the optimal allocation to retailer $i$ is given by $x_{i}^{*}=y_{i}(\theta)-v_{i}$. On the other hand, if $v_{i} \geq y_{i}(\theta)$, the initial inventory is greater than the target stock level and the retailer receives no allocation: $x_{i}^{*}=0$. Furthermore, as noted in Proposition 1, the value of $\theta$ for the equal fractile solution must satisfy the reserve stock constraint with equality:

$$
\sum_{\substack{i \in \mathcal{N} \\ y_{i}(\theta)-v_{i}>0}}\left(y_{i}(\theta)-v_{i}\right)=v_{0} .
$$




\subsection{Robust Optimization}

We now consider the same single period problem but using a robust optimization approach. Instead of random variables $\widetilde{\varepsilon}_{i}, i \in \mathcal{N}$, we consider a vector $\varepsilon$ belonging to an uncertainty set $U(\delta)$ defined by:

$$
U(\delta)=\left\{\varepsilon_{i}, i \in \mathcal{N}: \varepsilon_{i}<\delta\right\}
$$

for some parameter $\delta$. We also choose a target inventory level, $y_{i}$, for each location $i \in \mathcal{N}$. These targets specify a shipment policy, $x(y)$, as the solution to the following problem:

$$
S(y)=\min _{x} \sum_{i \in \mathcal{N}} x_{i}
$$

subject to

$$
\begin{aligned}
& x_{i} \geq y_{i}-v_{i}, \\
& x_{i} \geq 0,
\end{aligned}
$$

for each $i \in \mathcal{N}$. We also specify a weighting factor to apply to backorders at each retailer. Let $w_{i}$ denote the weight to apply to backorders at retailer $i, i \in \mathcal{N}$. A possible robust optimization model is to minimize the maximum weighted backorders across all retailers and all possible demand vectors:

$$
B^{*}=\min _{y_{i}, i \in \mathcal{N}, B} \max _{\varepsilon} B
$$

subject to

$$
\begin{aligned}
& B \geq w_{i}\left(\mu_{i}+\sigma_{i} \varepsilon_{i}-y_{i}\right) \\
& B \geq 0 \\
& \varepsilon_{i} \leq \delta
\end{aligned}
$$

for all $i \in \mathcal{N}$ and

$$
S(y) \leq v_{0}
$$

Note that the shipment optimization problem, $S(y)$, could be eliminated and its constraints incorporated into the backorder optimization problem directly. That is not possible in the multi-period formulations to follow; we maintain the separation at this point to emphasize the structure.

Proposition 2 If $v_{0}>0$, there exists an optimal robust solution satisfying, for each $i \in \mathcal{N}$,

$$
y_{i}^{*}=\mu_{i}+\sigma_{i} \delta-w_{i}^{-1} B^{*}
$$

Proof. Appendix 6.2.

Corollary 3 Let $y_{i}(B)=\mu_{i}+\sigma_{i} \delta-w_{i}^{-1} B$, for all $i \in \mathcal{N}$. Then, provided

$$
\sum_{i \in \mathcal{N}}\left(\mu_{i}+\sigma_{i} \delta-v_{i}\right)^{+}>v_{0}
$$


the optimal value of $B, B^{*}$, satisfies

$$
\sum_{i \in \mathcal{N}}\left(y_{i}\left(B^{*}\right)-v_{i}\right)^{+}=v_{0}
$$

The proposition implies that whereas the expected value criterion leads to a solution which, to the extent possible, equalizes the probability of a stockout across retailers, the robust optimization formulation leads to an equalization, to the extent possible, of maximum weighted backorders across retailers, where the maximum is relative to the worst case demand.

Consider the situation in which the backorder weighting factors, $w_{i}, i \in \mathcal{N}$, are inversely proportional to the corresponding standard deviations of demand, $\sigma_{i}, i \in \mathcal{N}:$

$$
w_{i}=\frac{w}{\sigma_{i}}
$$

for some constant $w$.

Corollary 4 If $w_{i}=\frac{w}{\sigma_{i}}$, for all $i \in \mathcal{N}$, and provided

$$
\sum_{i \in \mathcal{N}}\left(\mu_{i}+\sigma_{i} \delta-v_{i}\right)^{+}>v_{0}
$$

then the robust solution also minimizes $f_{1}(v)$.

Proof. Appendix 6.2.

That is, if the weights are inversely proportional to the standard deviations of demand and provided $\delta$ is not too small relative to the initial stock levels, the robust solution is identical to the expected value-minimizing solution.

\section{Multi-Period Stock Allocation}

In this section, we consider a multi-period version of the stock allocation problem. There are several periods of uncertain demand at the retailers and the warehouse has the opportunity to make costless allocations to the retailers at the beginning of each period, to the extent that it holds stock in reserve for these periods. As in the previous section, we begin by formulating the problem as an expected value minimization problem. No closed form solution to this problem exists but small restricted problems can be solved to optimality using linear programming. We then present a robust optimization formulation of the problem which will be the focus of the balance of the paper.

\subsection{Expected Value Optimization Formulation}

Let $T$ denote the stock allocation horizon, the number of periods considered in the stock allocation problem. Period $T+1$ is assumed to be a period in which the system is replenished with sufficient inventory from an external source to 
eliminate all backorders at all retailers. Let $t=1,2, \ldots, T$ index the periods in the stock allocation problem. The periods are not necessarily of equal length: As discussed earlier, it is often desirable to allow the first period to cover a longer span of time than the other periods because re-balancing of inventories does not typically become an issue until near the end of the horizon. We further assume that the lead time to ship from the warehouse to the retailers is negligible.

Let $\widetilde{d}_{i t}$ denote the random demand occuring at retailer $i$ during period $t$, and let $\widetilde{d}_{t}=\left(\widetilde{d}_{i t}\right)_{i \in \mathcal{N}}$ denote the vector of demands in period $t$. The stochastic process $\widetilde{d}=\left\{\widetilde{d}_{t}: t=1,2, \ldots, T\right\}$ is assumed to be independent from period-toperiod.

For a generic period $t$, let $v_{i}$ denote the beginning net inventory at retailer $i$, $i \in \mathcal{N}$, and let $v_{0}$ denote the stock held in reserve at the central warehouse. Let $v=\left(v_{0}, v_{1}, \ldots, v_{n}\right)$, as before. Again, we assume the cost of holding inventory is the same at all locations. We also assume that backorder costs are the same at all retailers. Our objective is to focus on allocation policies that have risk pooling as the predominate consideration. Let $f_{t}(v)$ denote the minimal expected total backorders over periods $t, t+1, \ldots, T$, given that the system begins period $t$ in state $(v)$. This function can be shown to satisfy the dynamic programming recursion given by:

$$
\begin{aligned}
& f_{T}(v)= \min _{\substack{x_{i} \geq 0, i \in \mathcal{N} \\
\sum_{i \in \mathcal{N}} x_{i} \leq v_{0}}} \sum_{i \in \mathcal{N}} E\left[\left(\widetilde{d}_{i t}-v_{i}-x_{i}\right)^{+}\right] ; \\
& f_{t}(v)=\min _{\substack{x_{i} \geq 0, i \in \mathcal{N} \\
\sum_{i \in \mathcal{N}} x_{i} \leq v_{0}}}\left\{\begin{array}{c}
\sum_{i \in \mathcal{N}} E\left[\left(\widetilde{d}_{i t}-v_{i}-x_{i}\right)^{+}\right] \\
+E\left[f_{t+1}\left(\left(v_{0}-\sum_{i \in \mathcal{N}} x_{i},\left(v_{i}+x_{i}-\widetilde{d}_{i t}\right)_{i \in \mathcal{N}}\right)\right)\right]
\end{array}\right\} ;
\end{aligned}
$$

for $t=1,2, \ldots, T-1$.

Computing $f_{1}(v)$ exactly is a severe computational challenge because of the so-called curse of dimensionality: the state space over which each $f_{t}$ must be evaluated is exponential in the number of retailers. As mentioned, past approaches in the literature have focussed on different approximation techniques to reduce the computational burden.

\subsection{Robust Optimization}

\subsubsection{The Risk Pooling Uncertainty Set}

Following the development of the one-period model, we define parameters $\mu_{i t}$ and $\sigma_{i t}$ to be the mean and standard deviation, respectively, of demand at retailer $i$ in period $t$, for each $i \in \mathcal{N}$, and $t=1,2, \ldots, T$. As before, the demand distribution at location $i$ in period $t$ is taken to be characterized by only these two parameters. Thus we assume that $d_{i t}=\mu_{i t}+\sigma_{i t} \varepsilon_{i t}$ where the variables $\varepsilon_{i t}$ are chosen from some uncertainty set $U(\delta)$, to be defined. We write the demand variables as $d_{i t}$, rather than $\widetilde{d}_{i t}$, because they are no longer interpreted as random variables. The uncertainty set contains constraints of the form $\varepsilon_{i t} \leq \delta$, for 
all $i$ and $t$; but, these constraints alone are insufficient to capture the motive for holding stock in reserve at the central warehouse. We require constraints that represent the phenomenon of risk pooling. For example, if we consider the sum of two independent, normalized demand variables, say $\widetilde{\varepsilon}_{i t}$ and $\widetilde{\varepsilon}_{j t}$ for $i \neq j$, then the standard deviation of the sum, $\widetilde{\varepsilon}_{i t}+\widetilde{\varepsilon}_{j t}$, is $\sqrt{2}$. An appropriate uncertainty set constraint on the sum would therefore be $\varepsilon_{i t}+\varepsilon_{j t} \leq \sqrt{2} \delta$. Extending this idea, we define the multi-period risk pooling uncertainty set $U(\delta)$ as follows:

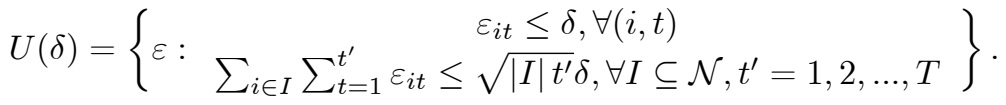

Since the number of sets satisfying $I \subseteq \mathcal{N}$ is $2^{N}$, this formulation of the risk pooling uncertainty set is impractical for large numbers of retailers. However, the problem of bounding the $k$ largest entries of an $n$-vector is known to have an equivalent formulation with fewer constraints. (See, for example, [Ben-Tal and Nemirovski, 2001], equation 18.c on p. 147. An alternative proof to the result below, using duality, can be found in [Zakeri et al., 2014]).

Lemma 5 The set $Z=\left\{z=\left(z_{i}\right)_{i \in \mathcal{N}}: \sum_{i \in I} z_{i} \leq M_{n}, \forall I \subseteq \mathcal{N},|I|=n\right\}$ for some $M_{n}, n \in \mathcal{N}$, is equivalent to the set $Z^{\prime}$ where

$$
Z^{\prime}=\left\{\begin{array}{c}
z=\left(z_{i}\right)_{i \in \mathcal{N}}: \exists \alpha_{n}, \beta_{n}=\left(\beta_{n i}\right)_{i \in \mathcal{N}} \text { s.t. } \\
n \alpha_{n}+\sum_{i=1}^{|\mathcal{N}|} \beta_{n i} \leq M_{n} \\
\alpha_{n}+\beta_{n i} \geq z_{i}, i \in \mathcal{N} ; \\
\beta_{i} \geq 0, i \in \mathcal{N} .
\end{array}\right\}
$$

Proof. If $z \in Z$, then order the elements of $z$ from largest, $z_{[1]}$, to smallest, $z_{[|\mathcal{N}|]}$. Let $\alpha_{n}=z_{[n]}$ and $\beta_{n i}=\left(z_{i}-\alpha_{n}\right)^{+}, i \in \mathcal{N}$. Let $I$ be the set of the $n$ largest elements of $z$. It is clear that $\beta_{n i}=0$ for all $i \notin I$. Since $z \in Z$ and $|I|=n$, we have

$$
\begin{aligned}
M_{n} & \geq \sum_{i \in I} z_{i} \\
& =n \alpha_{n}+\sum_{i \in I} \beta_{n i} \\
& =n \alpha_{n}+\sum_{i \in \mathcal{N}} \beta_{n i}
\end{aligned}
$$

establishing that $z \in Z^{\prime}$. Now, suppose $z \in Z^{\prime}$ and consider any subset $I$, $I \subseteq \mathcal{N}$ with $|I|=n$. Let $\left(\alpha_{n}, \beta_{n}\right)$ be any solution to the conditions in $Z^{\prime}$. Then, 
since $\beta_{n i} \geq 0$ for all $i$ :

$$
\begin{aligned}
M_{n} & \geq n \alpha_{n}+\sum_{i \in \mathcal{N}} \beta_{n i} \\
& \geq n \alpha_{n}+\sum_{i \in I} \beta_{n i} \\
& =\sum_{i \in I}\left(\alpha_{n}+\beta_{n i}\right) \\
& \geq \sum_{i \in I} z_{i} .
\end{aligned}
$$

Hence, $z \in Z$.

Observe that for a given $t^{\prime}, z_{i}$ can play the role of $\sum_{t=1}^{t^{\prime}} \varepsilon_{i t}$, and $M_{n}$ can play the role of $\sqrt{|I| t^{\prime}} \delta$ in (3) where $|I|=n$. Using this equivalent form of the risk pooling bound set leads to the following equivalent formulation of the risk pooling uncertainty set, $U(\delta)$ :

$$
U(\delta)=\left\{\begin{array}{c}
\varepsilon=\left(\varepsilon_{i t}\right)_{i \in \mathcal{N}, t \in T}: \\
\varepsilon_{i t} \leq \delta, i \in \mathcal{N}, t \in T ; \text { and } \\
\text { for all } n \in \mathcal{N}, t \in T: \\
\exists \alpha_{n t}, \beta_{n t}=\left(\beta_{n t i}\right)_{i \in \mathcal{N}} \text { s.t. } \\
n \alpha_{n t}+\sum_{i \in \mathcal{N}} \beta_{n t i} \leq \sqrt{n t} \delta \\
\alpha_{n t}+\beta_{n t i} \geq \sum_{t^{\prime}=1}^{t} \varepsilon_{i t^{\prime}}, \forall i \in \mathcal{N}, \\
\beta_{n t i} \geq 0, \forall i \in \mathcal{N} .
\end{array}\right\}
$$

Proposition 6 The number of constraints required to express the multi-period uncertainty set is of order $T N^{2}$ where $N$ is the number of retailers.

Proof. There are constraints in $U(\delta)$ for all combinations of $i \in \mathcal{N}, n \in \mathcal{N}$, and $t \in T$.

\subsubsection{Multi-Period Robust Optimization}

An Adaptive Shipment Policy Suppose the initial net inventory at location $i$ at the beginning of period 1 is given by $v_{i}, i \in \mathcal{N}$. The initial reserve stock at the central warehouse at the beginning of period 1 is $v_{0}$. The variable $y_{i t}$ represents the target net inventory level at retailer $i$ in period $t$, just after allocation from the central warehouse. The variable $x_{i t}$ represents the corresponding allocation from the central warehouse. The symbols $\mu, \sigma, v, d, \varepsilon, y$, and $x$ with subscripts omitted represent vectors of parameters or variables ranging over the omitted subscripts.

In this approach, the target net inventory levels, $y$, are chosen without reference to a particular $\varepsilon$ vector. The shipments in each period are allowed to be adaptive but they must be non-anticipatory. In particular, we require

$$
x_{i t}(\varepsilon)=\left(y_{i t}-v_{i}+\sum_{t^{\prime}=1}^{t-1}\left(\mu_{i t^{\prime}}+\sigma_{i t^{\prime}} \varepsilon_{i t^{\prime}}-x_{i t^{\prime}}(\varepsilon)\right)\right)^{+} .
$$


That is, in each period, the policy is to ship the minimum non-negative quantity required to achieve the target net inventory level, subject to past demands, shipments, and initial net inventories.

Lemma 7 Partial sums of required shipments satisfy the following relation:

$$
\sum_{t^{\prime}=1}^{t} x_{i t^{\prime}}(\varepsilon) \geq y_{i t}-v_{i}+\sum_{t^{\prime}=1}^{t-1}\left(\mu_{i t^{\prime}}+\sigma_{i t^{\prime}} \varepsilon_{i t^{\prime}}\right)
$$

with equality holding if $x_{i t^{\prime}}(\varepsilon)>0$.

Proof. By definition, $x_{i t}(\varepsilon) \geq\left(y_{i t}-v_{i}+\sum_{t^{\prime}=1}^{t-1}\left(\mu_{i t^{\prime}}+\sigma_{i t^{\prime}} \varepsilon_{i t^{\prime}}-x_{i t^{\prime}}(\varepsilon)\right)\right)$ with equality holding if $x_{i t}(\varepsilon)>0$. Rearranging terms yields the result.

Let $S(y, \varepsilon)$ denote the total shipments required over the stock allocation horizon following an adaptive, non-anticipatory policy for a given normalized demand vector, $\varepsilon$ :

$$
S(y, \varepsilon)=\sum_{i \in \mathcal{N}} \sum_{t=1}^{T}\left(y_{i t}-v_{i}+\sum_{t^{\prime}=1}^{t-1}\left(\mu_{i t^{\prime}}+\sigma_{i t^{\prime}} \varepsilon_{i t^{\prime}}-x_{i t^{\prime}}\right)\right)^{+} .
$$

Proposition 8 The total required shipment to support a target net inventory vector y given normalized demand vector $\varepsilon$ can be determined using the following linear program:

$$
S(y, \varepsilon)=\min \sum_{i \in \mathcal{N}} \sum_{t=1}^{T} x_{i t}
$$

subject to

$$
\begin{aligned}
\sum_{t^{\prime}=1}^{t} x_{i t^{\prime}}(\varepsilon) & \geq y_{i t}-v_{i}+\sum_{t^{\prime}=1}^{t-1}\left(\mu_{i t^{\prime}}+\sigma_{i t^{\prime}} \varepsilon_{i t^{\prime}}\right) \\
x_{i t} & \geq 0
\end{aligned}
$$

for all $i \in \mathcal{N}$ and $t=1,2, \ldots, T$.

Proof. Appendix 6.2.

Corollary 9 Alternatively,

$$
S(y, \varepsilon)=\max _{\pi} \sum_{i \varepsilon N} \sum_{t=1}^{T} \pi_{i t}\left(y_{i t}-v_{i}+\sum_{t^{\prime}=1}^{t-1}\left(\mu_{i t^{\prime}}+\sigma_{i t^{\prime}} \varepsilon_{i t^{\prime}}\right)\right)
$$

subject to

$$
\begin{aligned}
\sum_{t^{\prime}=t}^{T} \pi_{i t^{\prime}} & \leq 1 \\
\pi_{i t} & \geq 0
\end{aligned}
$$

for all $i \in \mathcal{N}$ and $t=1,2, \ldots, T$. 
Proof. The result follows easily by duality.

For each retailer $i \in \mathcal{N}$, let $t_{i}(\varepsilon)$ denote the following maximizing period for retailer $i$ :

$$
t_{i}(\varepsilon)=\arg \max _{t=1,2, \ldots, T}\left(y_{i t}-v_{i}+\sum_{t^{\prime}=1}^{t-1}\left(\mu_{i t^{\prime}}+\sigma_{i t^{\prime}} \varepsilon_{i t^{\prime}}\right)\right) .
$$

In the case of ties, select the earliest period which achieves the optimum. The resulting maximum may not be positive. Consequently, we let $t_{i}^{*}(\varepsilon)$ equal zero in such cases:

$$
t_{i}^{*}(\varepsilon)= \begin{cases}t_{i}(\varepsilon) & \text { if } y_{i t_{i}(\varepsilon)}+\sum_{t^{\prime}=1}^{t_{i}(\varepsilon)-1}\left(\mu_{i t^{\prime}}+\sigma_{i t^{\prime}} \varepsilon_{i t^{\prime}}\right)>v_{i} \\ 0 & \text { otherwise. }\end{cases}
$$

Proposition 10 The solution to the dual shipment problem, (6), is given by

$$
\pi_{i t}^{*}(\varepsilon)=1_{\left\{t=t_{i}^{*}(\varepsilon)\right\}}
$$

for all $i \in \mathcal{N}, t=1,2, \ldots, T$.

Proof. Appendix 6.2.

Let $\Pi$ denote the set

$$
\Pi=\left\{\pi: \sum_{t^{\prime}=1}^{T} \pi_{i t^{\prime}} \leq 1 ; \pi_{i t} \in\{0,1\} ; i \in \mathcal{N}, t=1,2, \ldots, T\right\} .
$$

Corollary 11 There exists an optimal solution to (9) satisfying $\pi \in \Pi$.

We can interpret the dual solution to the shipment problem as follows. Let $\tau_{i}(\varepsilon)$ denote the last period in which retailer $i$ receives a shipment, or zero if retailer $i$ receives no shipment:

$$
\tau_{i}(\varepsilon)= \begin{cases}\arg \max _{t=1,2, \ldots, T} 1_{\left\{x_{i t}(\varepsilon)>0\right\}} t, & \text { if } \sum_{t=1}^{T} x_{i t}(\varepsilon)>0 \\ 0, & \text { otherwise }\end{cases}
$$

Proposition 12 For a given target inventory vector $y$ and normalized demand vector $\varepsilon$, the shipment vector $x(\varepsilon)$ satisfies

$$
x_{i t}(\varepsilon)=0, \forall t>t_{i}^{*}(\varepsilon) .
$$

Furthermore, if $\tau_{i}(\varepsilon)>0$, then $t_{i}^{*}(\varepsilon)=\tau_{i}(\varepsilon)$.

Proof. Appendix 6.2.

Corollary 13 Barring ties, the vector $\pi$ optimizing (9) selects the last shipment period for each retailer. That is,

$$
\pi_{i t}(\varepsilon)=1_{\left\{t=\tau_{i}(\varepsilon)\right\}} .
$$


The Multi-Period Robust Stock Allocation Problem We say that a target net inventory vector $y$ is feasible if and only if its shipment requirements do not exceed the available reserve stock $v_{0}$ for any normalized demand vector in the uncertainty set. That is, $y$ is feasible iff

$$
S(y, \varepsilon) \leq v_{0}
$$

for all $\varepsilon \in U(\delta)$. Let $S(y)$ be the worst case shipping requirements:

$$
S(y)=\max _{\varepsilon \in U(\delta)} S(y, \varepsilon) .
$$

Then, $y$ is feasible iff

$$
S(y) \leq v_{0}
$$

Lemma $14 S(y)$ is non-decreasing in $y$.

Proof. For every $\varepsilon \in U(\delta)$, if $x$ is feasible for $y$, then it is also feasible for $y^{\prime} \leq y$. Hence, $S\left(y^{\prime}, \varepsilon\right) \leq S(y, \varepsilon)$ for all $\varepsilon \in U(\delta)$.

Let $w_{i t}$ denote the weight to apply to backorders at retailer $i$ in period $t$. Let $B_{t}$ denote the maximum weighted backorders in period $t$ across all retailers and all possible demand vectors. Our robust optimization formulation of the multiperiod stock allocation problem is to minimize the sum of maximum weighted backorders across retailers in each period and across all possible normalized demands, as follows:

$$
\min _{y, B} \sum_{t=1}^{T} B_{t}
$$

subject to, for all $\varepsilon \in U(\delta)$,

$$
\begin{aligned}
B_{t} & \geq w_{i t}\left(\mu_{i t}+\sigma_{i t} \varepsilon_{i t}-y_{i t}\right), \forall i \in \mathcal{N}, t=1, \ldots, T \\
B_{t} & \geq 0, t=1, \ldots, T \\
S(y) & \leq v_{0} .
\end{aligned}
$$

By definition of the risk pooling uncertainty set (3), for any $i \in \mathcal{N}$ and $t=$ $1, \ldots, T$, there exists a normalized demand vector $\varepsilon \in U(\delta)$ with $\varepsilon_{i t}=\delta$. (For example, set $\varepsilon_{j t^{\prime}}=0$ for all $\left(j, t^{\prime}\right) \neq(i, t)$.) Consequently, the problem reduces to

$$
\begin{aligned}
\min _{y, B} \sum_{t=1}^{T} B_{t} \\
B_{t} \geq w_{i t}\left(\bar{d}_{i t}(\delta)-y_{i t}\right), \forall i \in \mathcal{N}, t=1, \ldots, T ; \\
B_{t} \geq 0, t=1, \ldots, T ; \\
S(y) \leq v_{0},
\end{aligned}
$$

where $\bar{d}_{i t}(\delta)=\mu_{i t}+\sigma_{i t} \delta, i \in \mathcal{N}$. The uncertainty set $U(\delta)$ has been removed from all but the sub-problem, $S(y)$. 
Proposition 15 There exists an optimal solution, $\left(y^{*}, B^{*}\right)$, to the multi-period stock allocation problem (7) satisfying

$$
y_{i t}^{*}=\bar{d}_{i t}(\delta)-w_{i t}^{-1} B_{t}^{*}
$$

for all $i \in \mathcal{N}$, and $t=1,2, \ldots, T$.

Proof. Appendix 6.2.

By proposition 9, we can restate the shipment requirement as follows:

$$
S(y)=\max _{\varepsilon \in U(\delta)} \max _{\pi \in \Pi} \sum_{i \varepsilon N} \sum_{t=1}^{T} \pi_{i t}\left(y_{i t}-v_{i}+\sum_{t^{\prime}=1}^{t-1}\left(\mu_{i t^{\prime}}+\sigma_{i t^{\prime}} \varepsilon_{i t^{\prime}}\right)\right)
$$

This is a bilinear program (it involves the products $\pi_{i t} \varepsilon_{i t^{\prime}}$ ) but we show later that it can be reformulated as a mixed-integer linear program.

For any $\pi \in \Pi$, let $S_{\pi}(y)$ be given by

$$
\begin{aligned}
S_{\pi}(y)= & \max _{\varepsilon \in U(\delta)} \sum_{i \varepsilon N} \sum_{t=1}^{T} \pi_{i t}\left(y_{i t}-v_{i}+\sum_{t^{\prime}=1}^{t-1}\left(\mu_{i t^{\prime}}+\sigma_{i t^{\prime}} \varepsilon_{i t^{\prime}}\right)\right) \\
= & \sum_{i \varepsilon N} \sum_{t=1}^{T} \pi_{i t} y_{i t}-\sum_{i \varepsilon N} \sum_{t=1}^{T} \pi_{i t}\left(v_{i}-\sum_{t^{\prime}=1}^{t-1} \mu_{i t^{\prime}}\right) \\
& +\max _{\varepsilon \in U(\delta)} \sum_{i \varepsilon N} \sum_{t=2}^{T} \pi_{i t} \sum_{t^{\prime}=1}^{t-1} \sigma_{i t^{\prime}} \varepsilon_{i t^{\prime}} .
\end{aligned}
$$

Observe that $S_{\pi}(y)$ is an affine function of $y$ for each $\pi \in \Pi$. Furthermore,

$$
S(y)=\max _{\pi \in \Pi} S_{\pi}(y) .
$$

It follows that an equivalent formulation of the robust multi-period stock allocation problem (7) is as follows:

$$
\begin{gathered}
\min _{y, B} \sum_{t=1}^{T} B_{t} \\
B_{t} \geq w_{i t}\left(\bar{d}_{i t}(\delta)-y_{i t}\right), \forall i \in \mathcal{N}, t=1, \ldots, T ; \\
B_{t} \geq 0, t=1, \ldots, T ; \\
S_{\pi}(y) \leq v_{0}, \forall \pi \in \Pi .
\end{gathered}
$$

Since for each $\pi \in \Pi, S_{\pi}(y)$ is affine in $y$ and since $\Pi$ is a finite set, it follows that the robust multi-period stock allocation problem can be expressed as a linear program. We refer to this formulation, (11)-(14), as the Linear Form of the Robust Multi-Period Stock Allocation Problem. In general, $\Pi$ is a large set and the coefficients of the linearized shipment requirement (10) can be expensive to compute. In the general case, we propose a decomposition approach. However, in certain special cases it is possible to solve the Linear Form directly. We treat the general case first. 


\section{Solution Techniques}

\subsection{The General Case: A Decomposition Algorithm}

In this section, we apply Benders' decomposition approach to solve the general multi-period robust stock allocation problem. We begin by re-expressing the worst case shipment requirement $(9), \max _{\pi \in \Pi} S_{\pi}(y)$, as a mixed-integer linear program (MILP) in the following manner. We know that for each retailer $i$ there is a last period, $t_{i}^{*}(\varepsilon)$, in which it receives stock. For $t_{i}^{*}(\varepsilon)>0$, let $u_{i t}=0$ if $t=t_{i}^{*}(\varepsilon)$ for retailer $i$ and $u_{i t}=1$ otherwise. By corollary 13, we will have $u_{i t}=1-\pi_{i t}$ at the optimum. Let $u_{i 0}=0$ if no shipment is made to retailer $i$ during the time horizon and $u_{i 0}=1$ otherwise. This covers the situation when $t_{i}^{*}(\varepsilon)=0$, that is, when $v_{i}$ is sufficiently large that retailer $i$ requires no shipment to meet the target inventory levels, $y_{i t}$, for any $t=1,2, \ldots, T$. Let $S_{i}$ denote the total shipment required by retailer $i$ under the adaptive shipment policy. By lemma 7 and proposition $12, S_{i}$ is given by

$$
S_{i}= \begin{cases}y_{i t_{i}^{*}(\varepsilon)}-v_{i}+\sum_{t^{\prime}=1}^{t_{i}^{*}(\varepsilon)-1}\left(\mu_{i t^{\prime}}+\sigma_{i t^{\prime}} \varepsilon_{i t^{\prime}}\right) & \text { if } t_{i}^{*}(\varepsilon)>0 \\ 0 & \text { otherwise }\end{cases}
$$

for each retailer $i \in \mathcal{N}$.

Proposition 16 An equivalent MILP for determining the shipment requirement function is given by:

$$
S(y)=\max _{\varepsilon, u, S} \sum_{i \varepsilon N} S_{i}
$$

subject to

$$
\begin{aligned}
S_{i} & \leq M u_{i 0}, i \in \mathcal{N} \\
S_{i} & \leq y_{i t}-v_{i}+\sum_{t^{\prime}=1}^{t-1}\left(\mu_{i t^{\prime}}+\sigma_{i t^{\prime}} \varepsilon_{i t^{\prime}}\right)+M u_{i t}, i \in \mathcal{N}, t=1,2, \ldots, T ; \\
\sum_{t=0}^{T} u_{i t} & =T, i \in \mathcal{N} \\
u_{i t} & \in\{0,1\}, i \in \mathcal{N}, t=1,2, \ldots, T \\
\varepsilon & \in U(\delta)
\end{aligned}
$$

where $M$ is a sufficiently large number.

Proof. Appendix 6.2.

Corollary 17 At optimality for (9) and (15), $\pi_{i t}=1-u_{i t}$, for all $i \in \mathcal{N}$ and $t=1,2, \ldots, \mathcal{N}$. 


\begin{tabular}{ll}
\hline Input & $\left(\mu_{i t}, \sigma_{i t}, w_{i t}\right)_{i \in \mathcal{N}, t=1,2, \ldots, T} ; v_{0},\left(v_{i}\right)_{i \in \mathcal{N}} ;$ Assume $v_{0} \geq 0 ;$ \\
\hline Output & $\left(y_{i t}^{*}, B_{t}^{*}, \pi_{i t}^{*}\right)_{i \in \mathcal{N}, t=1,2, \ldots, T} ;$ \\
\hline Step & Set $k \leftarrow 0 ; \pi_{i t}^{0} \leftarrow 0, \forall i \in \mathcal{N}, t=1,2, \ldots, T ; \Pi^{0} \leftarrow\left\{\pi^{0}\right\} ; S_{\pi^{0}}(\cdot) \equiv 0 ;$ \\
\hline 0 & Solve the master problem $(21)-(24)$ using $\Pi^{k} ;$ Extract solution $y^{k}, B^{k}$ \\
\hline 1 & Solve MILP $(15)-(20)$ using $y^{k} ;$ Extract solution $S\left(y^{k}\right), u^{k}$, and $\varepsilon^{k}$. \\
\hline 2 & If $S\left(y^{k}\right) \leq v_{0}$ then stop; Set $\left(y^{*}, B^{*}, \pi^{*}\right) \leftarrow\left(y^{k}, B^{k}, \pi^{k}\right)$. \\
\hline 3 & Else set $\pi_{i t}^{k+1} \leftarrow 1-u_{i t}^{k}, \forall i \in \mathcal{N}, t=1,2, \ldots, T ;$ \\
\hline 4 & Using $(10)$ extract the coefficients of $S_{\pi^{k+1}}(y)$ from $S\left(y^{k}\right), y^{k}, \varepsilon^{k}, \pi^{k+1} ;$ \\
\hline 5 & Set $\Pi^{k+1} \leftarrow \Pi^{k} \cup\left\{\pi^{k}\right\} ; k \leftarrow k+1 ;$ \\
\hline 7 & Return to step 1. \\
\hline
\end{tabular}

Table 1: Benders' decomposition algorithm applied to the linear form of the robust multi-period stock allocation problem.

Applying Benders' approach, we state the master problem in linear form as

$$
\min _{y, B} \sum_{t=1}^{T} B_{t}
$$

subject to

$$
\begin{aligned}
B_{t} & \geq w_{i t}\left(\bar{d}_{i t}(\delta)-y_{i t}\right), \forall i \in \mathcal{N}, t=1, \ldots, T ; \\
B_{t} & \geq 0, t=1, \ldots, T ; \\
S_{\pi}(y) & \leq v_{0}, \forall \pi \in \Pi^{k}
\end{aligned}
$$

where $S_{\pi}(y)$ is the affine function given by (10) and $\Pi^{k}$ is some subset of the full set $\Pi$ as of iteration $k$ of the algorithm. The master problem can be solved as a linear program. Benders' decomposition algorithm as applied to this problem is described in Table 4.1.

Proposition 18 Benders' decomposition algorithm (Table 4.1) solves the Linear Form of the Robust Multi-Period Stock Allocation Problem (11) - (14).

Proof. Appendix 6.2.

\subsubsection{Large Numbers of Retailers: Regional Models}

In many applications, the number of retailers can be extremely large. Commercial applications involve thousands of retail locations; public health applications similarly have thousands of points of dispensing. Even with the approach described above, it would be impractical to model uncertainty sets which consider all possible demand combinations. Other modeling and approximation approaches would be required in such applications. It is outside the scope of this 
paper to consider or compare many such approaches: that might be best left to application-specific research. Such models should also capture the multi-echelon distribution structure that is likely present in such systems. Nevertheless, we propose one variation which increases the reach of the robust optimization approach.

In the case of large numbers of retailers, they will likely be organized into sales regions. Demand within these regions is also likely to exhibit correlation. Suppose we wish to capture risk pooling effects at the inter-regional level as well as at the intra-regional level. In that case, a natural modeling approach is to imagine that demand at a retailer has two sources of variability, a regional effect and a local effect. Let $\widetilde{\varepsilon}_{t}^{g}$ denote the regional variation as a mean-zero, variance-one random variable for region $g$ and let $\widetilde{\varepsilon}_{i t}$ denote the local variation as a mean-zero, variance-one random variable for retailer $i$ in period $t$. All such random variables are mutually independent. Let $\mathcal{N}_{g}$ denote the set of retailers within region $g$ and let $\mathcal{G}$ denote the set of regions. Demand at retailer $i$ in period $t$ is modeled as

$$
\widetilde{d}_{i t}=\mu_{i t}+\sigma_{t}^{g} \widetilde{\varepsilon}_{t}^{g}+\sigma_{i t} \widetilde{\varepsilon}_{i t},
$$

for each $i \in \mathcal{N}_{g}, g \in \mathcal{G}$, and $t=1, \ldots, T$, where $\sigma_{t}^{g}$ is the standard deviation of the regional variation for region $g$. Modeling demand in this way leads to consideration of an uncertainty set with the following form:

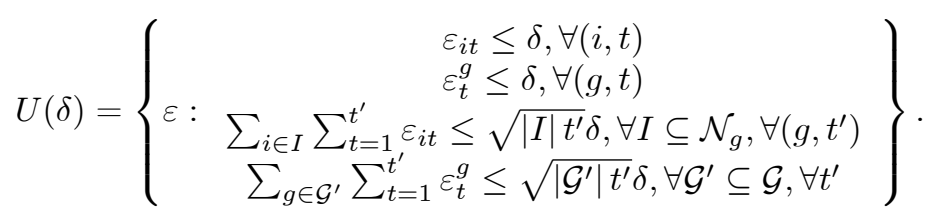

Observe that the approach of Lemma 5 to representing the uncertainty set constraints can be applied to each region separately as well as to the collection of regions. This drastically reduces the number of constraints needed.

We conclude that, with suitable approximations, the robust optimization approach is capable of capturing risk pooling effects even for large numbers of retailers.

\subsection{Special Cases}

\subsubsection{Special Case: No Initial Retail Inventories and Only Two Pe- riods}

Let us now consider the special case in which there is no initial stock at any retailer and there are only two periods in the planning horizon, that is, $v_{i}=0$ for all $i \in \mathcal{N}$, and $T=2$. In such cases, it is likely that each retailer will receive a shipment in the first period. The solution will therefore be indistinguishable from a situation in which initial retail inventories are non-zero but each retailer receives a shipment in the first period. Since the first period is typically associated with the receipt of stock at the central warehouse, this will be a common 
situation. In this case (10) simplifies to the following:

$$
S_{\pi}(y)=\max _{\varepsilon \in U(\delta)} \sum_{i \varepsilon N}\left(\pi_{i 1} y_{i 1}+\pi_{i 2} y_{i 2}+\pi_{i 2}\left(\mu_{i 1}+\sigma_{i 1} \varepsilon_{i 1}\right)\right) .
$$

Let $I_{1}=\left\{i: \pi_{i 1}=1\right\}$ and let $I_{2}=\left\{i: \pi_{i 2}=1\right\}$. By Corollary 13, $I_{1}$ is the set of retailers receiving their last shipment in the first period and $I_{2}$ is the set of retailers receiving a shipment in the second period. $S_{\pi}(y)$ is then the maximum shipment required to minimally achieve the target inventories $y$ under that arrangement across all normalized demand vectors in the uncertainty set. That is,

$$
S_{\pi}(y)=\sum_{i \in I_{1}} y_{i 1}+\sum_{i \in I_{2}} y_{i 2}+\sum_{i \in I_{2}} \mu_{i 1}+\max _{\varepsilon \in U(\delta)} \sum_{i \in I_{2}} \sigma_{i 1} \varepsilon_{i 1} .
$$

Consider the last term:

$$
\max _{\varepsilon \in U(\delta)} \sum_{i \in I_{2}} \sigma_{i 1} \varepsilon_{i 1} .
$$

Let $[j]$ index the retailer with the $j^{\text {th }}$ largest value of $\sigma_{i 1}$ for $i \in I_{2}$ and let $n_{2}=\left|I_{2}\right|$. Then,

$$
\sigma_{[1] 1} \geq \sigma_{[2] 1} \geq \cdots \geq \sigma_{\left[n_{2}\right] 1} .
$$

The optimization problem can be written as:

$$
\max \sum_{j=1}^{n_{2}} \sigma_{[j] 1} \varepsilon_{[j] 1}
$$

subject to

$$
\sum_{i \in I} \varepsilon_{i 1} \leq \sqrt{|I|} \delta, \forall I \subseteq I_{2}
$$

Proposition 19 An optimal solution to (26) is to set:

$$
\varepsilon_{[j] 1}=\delta(\sqrt{j}-\sqrt{j-1})
$$

for $j=1,2, \ldots, n_{2}$.

Proof. Appendix 6.2.

Corollary 20 If $\sigma_{i 1}=\sigma_{1}$ for all $i \in \mathcal{N}$, then

$$
\max _{\varepsilon \in U(\delta)} \sum_{i \in I_{2}} \sigma_{i 1} \varepsilon_{i 1}=\sqrt{\left|I_{2}\right|} \sigma_{1} \delta .
$$

Corollary 21 If $y \geq 0, \mu_{i 1}>0$, and $\pi$ is an optimal solution to Problem (9) for the given $y$, then $\pi_{i 1}+\pi_{i 2}=1$ for each $i \in \mathcal{N}$.

Proof. Appendix 6.2. 
Corollary 22 If $y \geq 0, \mu_{i 1}>0$, and $\pi$ is an optimal solution to Problem (9) for the given $y$, then $I_{1} \cup I_{2}=\mathcal{N}$ and $I_{1} \cap I_{2}=\varnothing$.

In an attempt to further simplify the problem, we assume, for the balance of this section, that $y \geq 0$ in the optimal solution to the robust stock allocation problem. This will likely be the case for sufficiently large values of the stock budget, $v_{0}$. By the corollary, we need only consider $\pi \in \Pi$ with $\pi_{i 1}+\pi_{i 2}=1, \forall i$.

Assembling these results, we have

$$
\max _{I_{2} \subseteq \mathcal{N}}\left\{\sum_{i \in \mathcal{N} \backslash I_{2}} y_{i 1}+\sum_{i \in I_{2}} y_{i 2}+\sum_{i \in I_{2}} \mu_{i 1}+\delta \sum_{j=1}^{\left|I_{2}\right|} \sigma_{[j] 1}(\sqrt{j}-\sqrt{j-1})\right\} \leq v_{0}
$$

where the selector function $[j]$ is relative to the chosen set $I_{2}$. Next, we exploit (8) to eliminate the $y$ vector from the formulation. The constraint $S(y) \leq v_{0}$ can be represented as

$$
\max _{I_{2} \subseteq \mathcal{N}}\left\{\begin{array}{c}
\sum_{i \in \mathcal{N} \backslash I_{2}}\left(\bar{d}_{i 1}(\delta)-w_{i 1}^{-1} B_{1}\right)+\sum_{i \in I_{2}}\left(\bar{d}_{i 2}(\delta)-w_{i 2}^{-1} B_{2}\right) \\
+\sum_{i \in I_{2}} \mu_{i 1}+\delta \sum_{j=1}^{\left|I_{2}\right|} \sigma_{[j] 1}(\sqrt{j}-\sqrt{j-1})
\end{array}\right\} \leq v_{0} .
$$

If we assume the retailers are identical in their demand characteristics and backorder penalties, then further simplication of the shipment constraint is possible, as we now show.

\subsubsection{Special Case: No Initial Retail Inventories, Two Periods, and Identical Retailers}

We continue the analysis of the two-period robust stock allocation problem when $v_{i}=0$, for all $i \in \mathcal{N}$ and $T=2$. In this section, we consider the special case in which the retailers are identical in their demand characteristics and weighting factors: $\mu_{i t}=\mu_{t}, \sigma_{i t}=\sigma_{t}$, and $w_{i t}=w_{t}$ for all $i \in \mathcal{N}$ and $t=1,2$. We continue to assume that $y \geq 0$ in the optimal solution to the robust stock allocation problem. In this case, for a relaxed version of the problem, it is possible to express the optimal solution in closed form.

Under the two-period, identical retailer case, let $n=\left|I_{2}\right|$, the number of retailers receiving a shipment in the second period. The two-period shipment constraint (29) simplifies to:

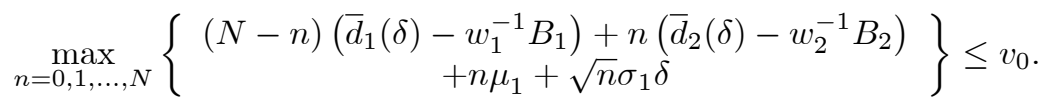

In particular, knowledge of the set of retailers receiving shipments in period 2 collapses to knowledge of just $n$, the number of retailers receiving shipment in period 2. The number of retailers receiving their last shipment in period 1 is therefore $(N-n)$. 
Let $y_{1}=\bar{d}_{1}(\delta)-w_{1}^{-1} B_{1}$ and $y_{2}=\bar{d}_{2}(\delta)-w_{2}^{-1} B_{2}$. We assume that $\delta$ is large enough to ensure $y \geq 0$. Substituting back into the linear form, and discarding constants from the objective function, results in a restatement of the robust stock allocation problem as

$$
\max _{y_{1}, y_{2}} w_{1} y_{1}+w_{2} y_{2}
$$

subject to

$$
\begin{aligned}
y_{t} & \leq \bar{d}_{t}(\delta), t=1,2 \\
(N-n) y_{1}+n y_{2}+n \mu_{1}+\sqrt{n} \sigma_{1} \delta & \leq v_{0}, n=1,2, \ldots, N .
\end{aligned}
$$

For the purpose of deriving analytical insight, we relax the integer constraint on $n$ and ignore the bounds on $y$ related to the maximum demand in each period. We designate the relaxed problem as the Relaxed Robust Two-Period Stock Allocation Problem with Identical Retailers (RR2), which is

$$
\max _{y_{1}, y_{2}} w_{1} y_{1}+w_{2} y_{2}
$$

subject to

$$
N y_{1}+\max _{1 \leq n \leq N}\left\{\sqrt{n} \sigma_{1} \delta-n\left(y_{1}-y_{2}-\mu_{1}\right)\right\} \leq v_{0} .
$$

Note that the maximand of the left hand side of (31) is a strictly concave function of $n$, provided $\sigma_{1} \delta>0$. Let $n^{*}$ denote its optimizing value in the optimal solution.

Theorem 23 If $N>\frac{w_{1}+w_{2}}{w_{2}}$ and $v_{0}>\frac{1}{2} \sigma_{1} \delta$, the solution to the problem (RR2) is given by:

$$
\begin{aligned}
& y_{1}^{*}=\frac{v_{0}}{N}-\frac{\left(\sigma_{1} \delta\right)}{2 \sqrt{\frac{w_{1}+w_{2}}{w_{2}} N}}, \\
& y_{2}^{*}=y_{1}^{*}-\mu_{1}-\frac{\sigma_{1} \delta}{2 \sqrt{\frac{w_{2}}{\left(w_{1}+w_{2}\right)} N}},
\end{aligned}
$$

and

$$
n^{*}=\frac{w_{2}}{\left(w_{1}+w_{2}\right)} N
$$

Proof. Let $D(y, n)$ denote the maximand of the left hand side of (31):

$$
D(y, n)=\sqrt{n} \sigma_{1} \delta-n\left(y_{1}-y_{2}-\mu_{1}\right) .
$$

Its partial derivative with respect to $n$ is given by

$$
\frac{\partial D(y, n)}{\partial n}=\frac{1}{2 \sqrt{n}} \sigma_{1} \delta-\left(y_{1}-y_{2}-\mu_{1}\right) .
$$


Setting this derivative to zero provides a formula for the unconstrained optimizer for $n$, denoted $\widehat{n}$ :

$$
\widehat{n}=\left(\frac{\sigma_{1} \delta}{2\left(y_{1}-y_{2}-\mu_{1}\right)}\right)^{2} .
$$

By the concavity of $D(y, \cdot)$,

$$
n^{*}=\left\{\begin{array}{cc}
1, & \text { if } y_{1}-y_{2}-\mu_{1}>\frac{1}{2} \sigma_{1} \delta \\
N, & \text { if } y_{1}-y_{2}-\mu_{1}<\frac{1}{2 \sqrt{N}} \sigma_{1} \delta \\
\widehat{n}, & \text { otherwise. }
\end{array}\right.
$$

Since the constraint (31) will be binding in an optimal solution, this results in three possible expressions for the optimal $y_{2}$ as a function of $y_{1}$ :

$$
y_{2}^{*}=\left\{\begin{array}{cc}
y_{1}-\mu_{1}+v_{0}-N y_{1}-\sigma_{1} \delta, & \text { if }\left(v_{0}-N y_{1}\right)<\frac{1}{2} \sigma_{1} \delta ; \\
\frac{v_{0}}{N}-\mu_{1}-\frac{1}{\sqrt{N}} \sigma_{1} \delta, & \text { if }\left(v_{0}-N y_{1}\right)>\frac{\sqrt{N}}{2} \sigma_{1} \delta \\
y_{1}-\mu_{1}+\frac{1}{\widehat{n}}\left(v_{0}-N y_{1}\right)-\frac{1}{\sqrt{\widehat{n}}} \sigma_{1} \delta, & \text { if } \frac{1}{2} \sigma_{1} \delta \leq\left(v_{0}-N y_{1}\right) \leq \frac{\sqrt{N}}{2} \sigma_{1} \delta .
\end{array}\right.
$$

Substituting for $\widehat{n}$ in the last expression, we see that if $\frac{1}{2} \sigma_{1} \delta \leq\left(v_{0}-N y_{1}\right) \leq$ $\frac{\sqrt{N}}{2} \sigma_{1} \delta$, then

$$
y_{2}^{*}=y_{1}-\mu_{1}+\frac{1}{\left(\frac{\sigma_{1} \delta}{2\left(y_{1}-y_{2}^{*}-\mu_{1}\right)}\right)^{2}}\left(v_{0}-N y_{1}\right)-\frac{1}{\left(\frac{\sigma_{1} \delta}{2\left(y_{1}-y_{2}^{*}-\mu_{1}\right)}\right)} \sigma_{1} \delta .
$$

Rearranging terms yields the following:

$$
0=\frac{4}{\left(\sigma_{1} \delta\right)^{2}}\left(y_{1}-y_{2}^{*}-\mu_{1}\right)^{2}\left(v_{0}-N y_{1}\right)-\left(y_{1}-y_{2}^{*}-\mu_{1}\right) .
$$

Simplifying, assuming $y_{2}^{*} \neq y_{1}-\mu_{1}$ :

$$
y_{2}^{*}=y_{1}-\mu_{1}-\frac{\left(\sigma_{1} \delta\right)^{2}}{4\left(v_{0}-N y_{1}\right)} .
$$

Let $Y=w_{1} y_{1}+w_{2} y_{2}$. Substituting for $y_{2}=y_{2}^{*}$,

$$
Y=\left\{\begin{array}{lc}
\left(w_{1}+w_{2}\right) y_{1}-w_{2} \mu_{1}+w_{2} v_{0}-w_{2} N y_{1}-w_{2} \sigma_{1} \delta, & \text { if }\left(v_{0}-N y_{1}\right)<\frac{1}{2} \sigma_{1} \delta ; \\
w_{1} y_{1}+w_{2} \frac{v_{0}}{N}-w_{2} \mu_{1}-\frac{w_{2}}{\sqrt{N}} \sigma_{1} \delta, & \text { if }\left(v_{0}-N y_{1}\right)>\frac{\sqrt{N}}{2} \sigma_{1} \delta ; \\
\left(w_{1}+w_{2}\right) y_{1}-w_{2} \mu_{1}-\frac{w_{2}\left(\sigma_{1} \delta\right)^{2}}{4\left(v_{0}-N y_{1}\right)}, & \text { if } \frac{1}{2} \sigma_{1} \delta \leq\left(v_{0}-N y_{1}\right) \\
& \leq \frac{\sqrt{N}}{2} \sigma_{1} \delta .
\end{array}\right.
$$

Taking the derivative of $Y$ with respect to $y_{1}$, we get

$$
\frac{d Y}{d y_{1}}=\left\{\begin{array}{cc}
w_{1}+w_{2}(1-N), & \text { if }\left(v_{0}-N y_{1}\right)<\frac{1}{2} \sigma_{1} \delta \\
w_{1}, & \text { if }\left(v_{0}-N y_{1}\right)>\frac{\sqrt{N}}{2} \sigma_{1} \delta \\
\left(w_{1}+w_{2}\right)-\frac{w_{2}\left(\sigma_{1} \delta\right)^{2} N}{4\left(v_{0}-N y_{1}\right)^{2}}, & \text { if } \frac{1}{2} \sigma_{1} \delta \leq\left(v_{0}-N y_{1}\right) \leq \frac{\sqrt{N}}{2} \sigma_{1} \delta .
\end{array}\right.
$$


Consequently, if $N>\frac{w_{1}+w_{2}}{w_{2}}$, any solution with $\left(v_{0}-N y_{1}\right)<\frac{1}{2} \sigma_{1} \delta$ can be improved by decreasing $y_{1}$. If, further, $v_{0}>\frac{1}{2} \sigma_{1} \delta$, then $y_{1}=0$ would dominate any solution in this region. Similarly, any solution with $\left(v_{0}-N y_{1}\right)>\frac{\sqrt{N}}{2} \sigma_{1} \delta$ could be improved by increasing $y_{1}$. The optimal $y_{1}$, therefore, must lie in the third region. Setting $\frac{d Y}{d y_{1}}=0$ in this region yields

$$
\left(v_{0}-N y_{1}^{*}\right)=\frac{1}{2} \sqrt{\frac{w_{2}}{\left(w_{1}+w_{2}\right)} N} \sigma_{1} \delta
$$

and solving for $y_{1}^{*}$ yields

$$
y_{1}^{*}=\frac{v_{0}}{N}-\frac{\left(\sigma_{1} \delta\right)}{2 \sqrt{\frac{w_{1}+w_{2}}{w_{2}} N}} .
$$

Substituting for $\left(v_{0}-N y_{1}^{*}\right)$ in the expression for $y_{2}^{*}$ yields

$$
\begin{aligned}
y_{2}^{*} & =y_{1}^{*}-\mu_{1}-\frac{\left(\sigma_{1} \delta\right)^{2}}{4\left(v_{0}-N y_{1}^{*}\right)} \\
& =y_{1}^{*}-\mu_{1}-\frac{\sigma_{1} \delta}{2 \sqrt{\frac{w_{2}}{\left(w_{1}+w_{2}\right)} N}} .
\end{aligned}
$$

Finally,

$$
\begin{aligned}
n^{*} & =\left(\frac{\sigma_{1} \delta}{2\left(y_{1}^{*}-y_{2}^{*}-\mu_{1}\right)}\right)^{2} \\
& =\left(\frac{\sigma_{1} \delta}{2\left(\frac{\sigma_{1} \delta}{\left.2 \sqrt{\frac{w_{2}}{\left(w_{1}+w_{2}\right)}}\right)}\right)}\right)^{2} \\
& =\frac{w_{2}}{\left(w_{1}+w_{2}\right)} N .
\end{aligned}
$$

Corollary 24 The optimal amount of stock to hold in reserve at the central warehouse at the end of period 1 is:

$$
v_{0}-N y_{1}^{*}=\frac{1}{2} \sqrt{\frac{w_{2}}{\left(w_{1}+w_{2}\right)} N} \sigma_{1} \delta
$$

Surprisingly, the optimal stock to hold in reserve at the central warehouse for allocation in period 2 in unaffected by $v_{0}$, the initial stock available in period 1 at the central warehouse, provided $v_{0}>\frac{1}{2} \sigma_{1} \delta$. To see this another way, Theorem 23 establishes that the worst case situation is for $\frac{w_{2}}{w_{1}+w_{2}} N$ retailers to receive shipments in the second period. This insight is new in the study of allocation problems. The total shipment required in period 2 is therefore 
$\frac{w_{2}}{w_{1}+w_{2}} N\left(y_{2}^{*}-y_{1}^{*}\right)$ plus the demand which can occur at these $\frac{w_{2}}{w_{1}+w_{2}} N$ retailers. Ignoring the integrality restriction on the number of retailers, this demand is at most

$$
\begin{aligned}
& \frac{w_{2}}{w_{1}+w_{2}} N \mu_{1}+\max _{\substack{\varepsilon \in U(\delta) \\
I_{2} \subseteq N \\
\left|I_{2}\right|=\frac{w_{2}}{w_{1}+w_{2}}}} \sum_{i \in I_{2}} \sigma_{1} \varepsilon_{i 1} \\
= & \frac{w_{2}}{w_{1}+w_{2}} N \mu_{1}+\sqrt{\frac{w_{2}}{w_{1}+w_{2}} N} \sigma_{1} \delta,
\end{aligned}
$$

by Corollary 20 in the identical retailer case. The stock held in reserve for period 2 must therefore be at least

$$
\begin{aligned}
& \frac{w_{2}}{w_{1}+w_{2}} N\left(y_{2}^{*}-y_{1}^{*}\right)+\frac{w_{2}}{w_{1}+w_{2}} N \mu_{1}+\sqrt{\frac{w_{2}}{w_{1}+w_{2}} N} \sigma_{1} \delta \\
= & \frac{w_{2}}{w_{1}+w_{2}} N\left(y_{2}^{*}-y_{1}^{*}+\mu_{1}\right)+\sqrt{\frac{w_{2}}{w_{1}+w_{2}} N} \sigma_{1} \delta \\
= & \frac{w_{2}}{w_{1}+w_{2}} N\left(\frac{-\sigma_{1} \delta}{2 \sqrt{\frac{w_{2}}{\left(w_{1}+w_{2}\right)}}}\right)+\sqrt{\frac{w_{2}}{w_{1}+w_{2}} N} \sigma_{1} \delta \\
= & \frac{1}{2} \sqrt{\frac{w_{2}}{w_{1}+w_{2}} N} \sigma_{1} \delta
\end{aligned}
$$

units, as the corollary states.

Corollary 25 If $N>2, w_{1}=w_{2}$, and $v_{0}>\frac{1}{2} \sigma_{1} \delta$, the solution to the problem $(R R 2)$ is given by:

$$
\begin{aligned}
& y_{1}^{*}=\frac{v_{0}}{N}-\frac{\left(\sigma_{1} \delta\right)}{2 \sqrt{2 N}}, \\
& y_{2}^{*}=y_{1}^{*}-\mu_{1}-\frac{\left(\sigma_{1} \delta\right)}{\sqrt{2 N}},
\end{aligned}
$$

and

$$
n^{*}=N / 2 .
$$

and the optimal stock to hold in reserve at the central warehouse for period 2 in problem $(R R 2)$ is given by

$$
v_{0}-N y_{1}^{*}=\frac{1}{2} \sqrt{\frac{N}{2}} \sigma_{1} \delta .
$$

Another way to view this result is to observe that the stock employed for pooling risk in this allocation problem, $v_{0}-N y_{1}^{*}$, grows with the square root of the number of retailers. Another insight is that $y_{2}^{*}$ approaches $y_{1}^{*}-\mu_{1}$ as $N \rightarrow \infty$. That is, due to risk pooling, the target minimum inventory level for each retailer in period 2 becomes less dependent on demand variability as the number of retailers increases. 


\section{Experimental Results}

In this section we report on an empirical study conducted to assess the contribution robust optimization can make to optimizing stock allocation problems. A general scheme for generating test cases is detailed in Appendix 6.1. The relevant parameters are as follows:

- $N$, the number of retailers;

- $T$, the number of time periods (opportunities for allocation) in the cycle;

- $\bar{\mu}$, the average demand per retailer per day;

- $\bar{l}$, the average number of days per period;

- $\psi$, the coefficient of variation of daily demand of the smallest retailer (larger retailers experience lower coefficients of variation; hence $\psi$ is the maximum coefficient of variation across retailers);

- $\beta_{D}$, the Pareto demand shape parameter $\left(\beta_{D}=0.2\right.$ results in identically distributed demands across retailers, and $\beta_{D}=0.8$ results in an 80-20 distribution: $80 \%$ of expected demand comes from only $20 \%$ of the retailers);

- $\beta_{L}$, the Pareto period-length shape parameter $\left(\beta_{L}=0.2\right.$ results in equal period lengths, and $\beta_{L}=0.8$ results in an 80-20 distribution: $80 \%$ of total cycle days are concentrated in the first $20 \%$ of the number of periods); and

- $\gamma$, the number of standard deviations of total system demand to hold as safety stock.

The shape parameters allow us to generate test cases with non-identical retailers and unequal period lengths. The appendix also details a method for generating backorder weights but none of the experiments conducted to date employ non-identical weights. For the purpose of this section, then, we take $w_{i t}=1$ for all $i \in \mathcal{N}$ and all $t=1,2, \ldots, T$. Table 2 summarizes the parameters used to generate test cases.

Demands are simulated using truncated normals. That is, we set

$$
\widetilde{d}_{i t}=\left(\mu_{i t}+\sigma_{i t} \widetilde{\varepsilon}_{i t}\right)^{+}
$$

for each $i \in \mathcal{N}$ and all $t=1,2, \ldots, T$, where each $\widetilde{\varepsilon}_{i t}$ is drawn independently from a $N(0,1)$ distribution. Note, first, that these demands cover allocation periods each of which may span several demand periods. For example, if the first allocation period covers five demand days, then $\widetilde{d}_{i 1}$ represents five days of demand. Note, second, that the demand truncation introduces bias into the simulation (i.e. $E\left[\widetilde{d}_{i t}\right]>\mu_{i t}$ ) and our results must be interpreted in light of this bias. Each policy considered faces the same bias and so comparisons between feasible policies should still be valid. However, in the case of non-identical 


\begin{tabular}{lll}
\hline Parameter Name & Symbol & Value Range \\
\hline Number of retailers & $N$ & 4,8 \\
\hline Number of periods & $T$ & 2,3 \\
\hline Mean daily demand & $\bar{\mu}$ & $2.5,5$ \\
\hline Mean days per period & $\bar{l}$ & 5 \\
\hline Coefficient of variation & $\psi$ & $0.5,1,1.5,2,2.5,3$ \\
\hline Pareto demand shape & $\beta_{D}$ & $0.2,0.8$ \\
\hline Pareto period-length shape & $\beta_{L}$ & $0.2,0.8$ \\
\hline Safety stock factor & $\gamma$ & $1,1.5,2,2.5$ \\
\hline
\end{tabular}

Table 2: Parameter ranges for generating test cases.

retailers, the lower bounding policy may not yield a true lower bound as a result of this bias. Most of the results, however, refer to the identical retailer case.

Each simulation run corresponds to a sample vector $\widetilde{\varepsilon}^{k}$, given by

$$
\widetilde{\varepsilon}^{k}=\left(\widetilde{\varepsilon}_{i t}^{k}\right)_{(i, t) \in \mathcal{N} \times\{1,2, \ldots, T\}}
$$

for sample index $k$. We generate a total of 10,000 sample vectors (or cycles) and re-use the same sample set with each experiment. This is a variance reduction technique allowing us to estimate more precisely the impact of different policies on performance. Each experiment is divided into 10 sample groups with $K=$ 1000 sample vectors in each group. Performance metrics are computed for each sample group and then averaged over all groups.

We simulate the performance of different policies in a rolling horizon setting and compare them under different metrics. For simplicity, we assume that initial inventories at all retailers are zero: $v_{i}=0$, for all $i \in \mathcal{N}$. Let $\widetilde{x}^{k P}=\left(\widetilde{x}_{i t}^{k P}\right)$ denote the shipment decisions made under generic policy $P$ for sample vector $k$. Two metrics considered for each sample group are average time-weighted backorders:

$$
B^{P}=\frac{1}{K} \sum_{k=1}^{K} \sum_{i \in \mathcal{N}} \sum_{t=1}^{T}\left(\sum_{t^{\prime}=1}^{t} \widetilde{d}_{i t^{\prime}}^{k}-\sum_{t^{\prime}=1}^{t} \widetilde{x}_{i t^{\prime}}^{k P}\right)^{+}
$$

and average terminal backorders:

$$
B_{T}^{P}=\frac{1}{K} \sum_{k=1}^{K} \sum_{i \in \mathcal{N}}\left(\sum_{t^{\prime}=1}^{T} \widetilde{d}_{i t^{\prime}}^{k}-\sum_{t^{\prime}=1}^{T} \widetilde{x}_{i t^{\prime}}^{k P}\right)^{+} .
$$

Other metrics are introduced below for specific policies.

\subsection{Robust Allocation in a Rolling Horizon}

The Linear Form of the Robust Multi-Period Stock Allocation Problem is given by (11)-(14). Implicit in the definition of the shipment requirement function, 
$S_{\pi}(y)$, is the vector of initial inventories $v$. The robust allocation policy is formed by re-solving the robust multi-period stock allocation problem at the beginning of each period, setting the problem data to match the remaining periods of allocation and the initial inventories to match the residual net inventories at the end of the previous period. The Robust policy is denoted as $\widetilde{x}^{R}$ and its computed average time-weighted backorders as $B^{R}$ and average terminal backorders as $B_{T}^{R}$.

\subsection{Policy Bounds}

A lower bound on the minimal number of expected backorders can be developed by allowing costless, instantaneous re-balancing of retail inventories in each period. Suppose, in each period, echelon inventory is instantaneously reallocated without restriction among retailers. We refer to this as the Rebalance policy and denote it with a superscript $B$. If $v_{t}$ is the total echelon net inventory at the beginning of period $t$, then the optimal allocation in period $t$, under the balance assumption, is a myopic policy given by $x_{t}^{B}\left(v_{t}\right)$ :

$$
x_{t}^{B}\left(v_{t}\right)=\arg \min _{\substack{x_{i} \geq 0, i \in \mathcal{N} \\ \sum_{i \in \mathcal{N}} x_{i} \leq v_{t}}} \sum_{i \in \mathcal{N}} E\left[\left(\widetilde{d}_{i t}-x_{i}\right)^{+}\right] .
$$

We denote the average time-weighted backorders for this policy by $B^{B}$ and the average terminal backorders by $B_{T}^{B}$. The Rebalance policy is not feasible for the system we consider. However, it is easy to see that it yields a lower bound on our backorder metrics.

A natural upper bound on backorder metrics can be found by following the Eppen and Schrage Ship All policy in which all stock is allocated in the first period and no stock is held in reserve for rebalancing purposes. As noted, this policy can be seen as a consequence of assuming that inventory rebalancing is possible in every period. With that assumption, together with our assumption that inventory holding costs are identical at every location, there is no motive for holding inventory at the central warehouse. We use a superscript $A$ to denote the Ship All policy:

$$
\widetilde{x}_{1}^{A}=\arg \min _{\substack{x_{i 1} \geq 0, i \in \mathcal{N} \\ \sum_{i \in \mathcal{N}} x_{i 1} \leq v_{0}}} \sum_{i \in \mathcal{N}} E\left[\left(\sum_{t^{\prime}=1}^{T} \widetilde{d}_{i t^{\prime}}-s_{i}-x_{i}\right)^{+}\right]
$$

and $\widetilde{x}_{i t}^{A}=0$, for all $i \in N$ and $t>1$. Since this policy does not reserve any stock at the central warehouse, it cannot capture any of the potential risk-pooling. We denote the corresponding average time-weighted backorders for this policy by $B^{A}$ and the average terminal backorders by $B_{T}^{A}$. Also note that our bounds are sensitive to the number of allocation periods, $T$, and their lengths.

It will be seen that, for some experiments, the Ship All policy outperforms the Robust policy with respect to average time-weighted backorders. The Robust policy, on the other hand, will be seen to always outperform the Ship All 
policy with respect to average terminal backorders. However, this latter comparison is not fair. If the objective were truly to minimize expected terminal backorders, it is easy to see that the optimal policy would be to reserve all stock at the central warehouse until the last period, after demands for all periods except the last period have been observed, and then to allocate it to minimize expected terminal backorders. This suggests that the Robust policy may be holding an excessive amount of stock in reserve.

To test this conjecture, we also consider a simple Ship Mean policy which sets the target inventory level at each retailer in each period, except the last, equal to the expected demand at that retailer in that period. That is, shipments are made to raise net inventory at each retailer to the mean demand for the next period. In the last period, or in any period for which central stock is insufficient to meet the targets, the policy reverts to the Ship All policy in that period, allocating stock to minimize expected terminal backorders in one final set of shipments. Because the Ship Mean policy is so conservative in holding stock in reserve until the last period, it can be expected to outperform other feasible policies with respect to average terminal backorders but perform poorly with respect to average time-weighted backorders. This comparison will permit us to better see where the Robust policy falls between these two extremes. We denote the average time-weighted backorders for the Ship Mean policy by $B^{M}$ and the average terminal backorders by $B_{T}^{M}$.

Since the Ship All policy involves no risk-pooling and the Rebalance policy captures all possible risk-pooling, the differences in metrics, $B^{A}-B^{B}$ and $B_{T}^{A}-B_{T}^{B}$, are upper bounds on the risk-pooling benefit (average time-weighted backorders and average terminal backorders, resp.) possible from holding some stock in reserve at the central warehouse. Recall $B^{R}\left(B_{T}^{R}\right.$, resp.) represent the average time-weighted backorders (average terminal backorders, resp.) when following the Robust policy. For each experiment sample group, we score the Robust policy using percentages

$$
C^{R}=\frac{B^{A}-B^{R}}{B^{A}-B^{B}} \times 100 \%
$$

and

$$
C_{T}^{R}=\frac{B_{T}^{A}-B_{T}^{R}}{B_{T}^{A}-B_{T}^{B}} \times 100 \%,
$$

called the capture percentage and terminal capture percentage, respectively. We score the Ship Mean policy in a similar fashion (substitute $B^{M}$ for $B^{R}$ and $B_{T}^{M}$ for $B_{T}^{R}$ ), denoting the results by $C^{M}$ and $C_{T}^{M}$, respectively. Using the 10 sample groups for each experiment we construct $95 \%$ confidence intervals around the mean capture percentages and mean terminal capture percentages.

Since the test cases differ widely, we need a metric to compare the ease or difficulty of the underlying allocation problem. Let $D_{T}$ denote the average total demand in a sample group:

$$
D_{T}=\frac{1}{K} \sum_{k=1}^{K} \sum_{i \in \mathcal{N}} \sum_{t^{\prime}=1}^{T} \widetilde{d}_{i t^{\prime}}^{k} .
$$


Then a measure of problem ease is given by $F_{T}^{B}$ :

$$
F_{T}^{B}=\left(1-\frac{B_{T}^{B}}{D_{T}}\right) \times 100 \%
$$

which we call the Rebalance terminal fill rate: the fraction of demands served within the allocation cycle under the Rebalance policy. The lower this ideal fill rate is, the more difficult the underlying allocation problem must be. We also compute terminal fill rates for the Ship All policy:

$$
F_{T}^{A}=\left(1-\frac{B_{T}^{A}}{D_{T}}\right) \times 100 \%
$$

and for the Robust policy:

$$
F_{T}^{R}=\left(1-\frac{B_{T}^{R}}{D_{T}}\right) \times 100 \%
$$

As with the other metrics, we use the 10 sample groups to estimate means and confidence intervals for $F_{T}^{B}, F_{T}^{A}$, and $F_{T}^{R}$. As with the other bounds, the fill rate statistics are sensitive to the number of allocation periods and their lengths. In particular, the fill rate for the Ship All policy varies with $T$ and the length of the allocation periods.

\subsection{Test Results}

Unless otherwise noted, the number of retailers, $N$, is 5 , the number of allocation periods, $T$, is 2 , the average daily demand per retailer, $\bar{\mu}$, is 5 , the average period length, $\bar{l}$, is 5 , and the safety stock factor, $\gamma$, is 2 . We explore other values for these parameters in some cases as well.

\subsubsection{Identical Retailers, Equal Period Lengths, Varying COVs}

In this set of experiments, we consider identical retailers $\left(\beta_{D}=0.2\right)$ with equal period lengths $\left(\beta_{L}=0.2\right)$ and we vary the coefficient of variation of daily retailer demand, $\psi$. Table 3 shows the results. We notice immediately that the Robust policy is capturing a large share of the risk pooling potential ranging from $60.7 \%$ to $86.2 \%$. Ignoring time-weighted backorders, we see that the Robust policy captures between $78.2 \%$ to $100 \%$ of risk pooling potential. These capture percentages decrease markedly with increases in the coefficient of variation. Not surprisingly, we see that the rebalance terminal fill rate also decreases with increases in the coefficient of variation, dropping from $99.4 \%$ to $96.48 \%$, indicating that the difficulty of the underlying allocation problem is increasing in the coefficient of variation.

\subsubsection{Identical Retailers, Pareto Period Lengths, Varying COVs}

In this set of experiments, we consider identical retailers and varying coefficients of variation, but we make the first period longer than the second period by 


\begin{tabular}{|l|c|c|c|c|c|}
\hline$\psi, \mathbf{C O V}$ & $C^{R}$ & $\mathbf{C}_{T}^{R}$ & $\mathbf{F}_{T}^{B}$ & $\mathbf{F}_{T}^{A}$ & $\mathbf{F}_{T}^{R}$ \\
\hline \multirow{2}{*}{0.5} & 86.2 & 100.0 & 99.40 & 98.72 & 99.40 \\
& \pm 1.0 & \pm 0.0 & \pm 0.03 & \pm 0.04 & \pm 0.03 \\
\hline \multirow{2}{*}{1.0} & 85.8 & 99.6 & 98.80 & 97.44 & 98.79 \\
& \pm 1.1 & \pm 0.5 & \pm 0.05 & \pm 0.08 & \pm 0.06 \\
\hline \multirow{2}{*}{1.5} & 82.0 & 95.0 & 98.19 & 96.22 & 98.09 \\
& \pm 1.7 & \pm 1.2 & \pm 0.08 & \pm 0.12 & \pm 0.09 \\
\hline \multirow{2}{*}{2.0} & 73.3 & 84.4 & 97.59 & 95.12 & 97.20 \\
& \pm 2.0 & \pm 1.6 & \pm 0.10 & \pm 0.15 & \pm 0.11 \\
\hline \multirow{2}{*}{2.5} & 64.6 & 76.5 & 97.01 & 94.16 & 96.34 \\
& \pm 2.1 & \pm 1.8 & \pm 0.12 & \pm 0.18 & \pm 0.13 \\
\hline \multirow{2}{*}{3.0} & 60.7 & 78.2 & 96.48 & 93.32 & 95.79 \\
& \pm 2.1 & \pm 1.8 & \pm 0.14 & \pm 0.20 & \pm 0.14 \\
\hline
\end{tabular}

Table 3: Simulation results for identical retailers, equal period lengths, and varying coefficients of variation, showing robust capture percentage, $C^{R}$, robust terminal capture percentage, $C_{T}^{R}$, and terminal fill rates, $F_{T}^{B}, F_{T}^{A}$, and $F_{T}^{R}$.

setting $\beta_{L}=0.8$. As a result, the first period is 8 days in length and the second period is 2 days in length. As motivated in the appendix, we refer to this setting as Pareto period lengths.

Table 4 shows the results. The results initially suggest that shifting the allocation point to later in the cycle makes things worse: the robust capture percentages have all decreased from their counterparts in Table 3. Notice, however, that the robust terminal fill rates are all higher as a result of the relative change in period lengths. As first noted by Silver, this suggests that shifting the allocation point to later in the cycle makes it easier to achieve high service levels. Put another way, the opportunity to exploit risk pooling has increased with this change in period lengths. In this light, the Robust policy is capturing a smaller share of a larger pie.

\subsubsection{Identical Retailers, Three Periods}

In this section, we return to considering identical retailers, equal length periods, and varying coefficients of variation; but we extend the number of periods to $T=3$.

Table 5 shows the results for this three period case and we compare the results with Table 3 . We note first that the rebalance terminal fill rate is generally higher for the three-period case than for the two-period case, suggesting that the opportunities for risk pooling are greater. The Robust policy terminal capture percentage is also generally higher for the three-period case than for the twoperiod case. It is therefore capturing a larger share of a larger pie. The story is mixed for the robust capture percentage: in the three-period test case the capture percentage is lower $(82.9 \%)$ for the lowest coefficient of variation than in the two-period case (86.2\%) but the reverse is true for the highest coefficient 


\begin{tabular}{|l|c|c|c|c|c|}
\hline$\psi, \mathbf{C O V}$ & $C^{R}$ & $\mathbf{C}_{T}^{R}$ & $\mathbf{F}_{T}^{B}$ & $\mathbf{F}_{T}^{A}$ & $\mathbf{F}_{T}^{R}$ \\
\hline \multirow{2}{*}{0.5} & 75.6 & 84.3 & 99.77 & 98.72 & 99.60 \\
& \pm 1.4 & \pm 1.2 & \pm 0.01 & \pm 0.05 & \pm 0.02 \\
\hline \multirow{2}{*}{1.0} & 48.6 & 75.5 & 99.54 & 97.44 & 99.02 \\
& \pm 1.2 & \pm 1.3 & \pm 0.03 & \pm 0.11 & \pm 0.05 \\
\hline \multirow{2}{*}{1.5} & 33.8 & 75.8 & 99.31 & 96.16 & 98.55 \\
& \pm 1.5 & \pm 1.3 & \pm 0.04 & \pm 0.16 & \pm 0.07 \\
\hline \multirow{2}{*}{2.0} & 25.3 & 76.2 & 99.10 & 94.96 & 98.11 \\
& \pm 1.7 & \pm 1.3 & \pm 0.05 & \pm 0.20 & \pm 0.09 \\
\hline \multirow{2}{*}{2.5} & 20.0 & 76.7 & 98.90 & 93.88 & 97.72 \\
& \pm 1.7 & \pm 1.3 & \pm 0.06 & \pm 0.23 & \pm 0.11 \\
\hline \multirow{2}{*}{3.0} & 16.5 & 77.3 & 98.70 & 92.92 & 97.38 \\
& \pm 1.8 & \pm 1.2 & \pm 0.07 & \pm 0.26 & \pm 0.12 \\
\hline
\end{tabular}

Table 4: Simulation results for identical retailers, Pareto period lengths, and varying coefficients of variation, showing robust capture percentage, $C^{R}$, robust terminal capture percentage, $C_{T}^{R}$, and terminal fill rates, $F_{T}^{B}, F_{T}^{A}$, and $F_{T}^{R}$.

\begin{tabular}{|l|c|c|c|c|c|}
\hline$\psi, \mathbf{C O V}$ & $C^{R}$ & $\mathbf{C}_{T}^{R}$ & $\mathbf{F}_{T}^{B}$ & $\mathbf{F}_{T}^{A}$ & $\mathbf{F}_{T}^{R}$ \\
\hline \multirow{2}{*}{0.5} & 82.9 & 100.0 & 99.68 & 98.94 & 99.68 \\
& \pm 1.1 & \pm 0.0 & \pm 0.02 & \pm 0.03 & \pm 0.02 \\
\hline \multirow{2}{*}{1.0} & 82.4 & 99.3 & 99.36 & 97.87 & 99.34 \\
& \pm 1.2 & \pm 0.4 & \pm 0.04 & \pm 0.06 & \pm 0.03 \\
\hline \multirow{2}{*}{1.5} & 79.6 & 95.5 & 99.01 & 96.85 & 98.91 \\
& \pm 1.3 & \pm 0.8 & \pm 0.05 & \pm 0.08 & \pm 0.05 \\
\hline \multirow{2}{*}{2.0} & 77.1 & 92.2 & 98.63 & 95.90 & 98.42 \\
& \pm 1.1 & \pm 0.8 & \pm 0.07 & \pm 0.10 & \pm 0.07 \\
\hline \multirow{2}{*}{2.5} & 75.2 & 89.9 & 98.24 & 95.02 & 97.92 \\
& \pm 1.0 & \pm 1.0 & \pm 0.09 & \pm 0.12 & \pm 0.08 \\
\hline \multirow{2}{*}{3.0} & 73.1 & 87.7 & 97.86 & 94.22 & 97.41 \\
& \pm 1.0 & \pm 1.2 & \pm 0.10 & \pm 0.14 & \pm 0.10 \\
\hline
\end{tabular}

Table 5: Simulation results for identical retailers, three equal-length periods, and varying coefficients of variation, showing robust capture percentage, $C^{R}$, robust terminal capture percentage, $C_{T}^{R}$, and terminal fill rates, $F_{T}^{B}, F_{T}^{A}$, and $F_{T}^{R}$. 
of variation ( $73.1 \%$ versus $60.7 \%)$.

Comparing the results with Table 4, we note that the risk pooling opportunities are greater with the two-period Pareto-length case than for the three equal periods case (the rebalance terminal fill rates are generally higher). However, the differences are not as pronounced as with Table 3. On the other hand, the capture percentages are much lower. A tentative conclusion is that given a choice between two regimes for allocating stock: three equal periods versus two-periods with Pareto-lengths, the Robust policy will fare better under the three equal-period regime.

\subsubsection{Lower Demand Rate}

In this set of experiments, we consider identical retailers, two equal-length periods, and varying coefficients of variation, but we cut the demand rate in half, $\bar{\mu}=2.5$, to see if the results are sensitive to scale. The results are not displayed as they are identical to Table 3. This is not surprising because the coefficient of variation is preserved through the scaling.

\subsubsection{Identical Retailers, Two Pareto-Periods, Varying Safety Stock Factor}

In this set of experiments, we consider the impact of the safety stock factor and the choice of the uncertainty set parameter. All the experiments are run with identical retailers $\left(\beta_{D}=0.2\right)$, two Pareto-periods $\left(\beta_{L}=0.8\right)$, and a high coefficient of variation $(\psi=3)$. From Table 4 , we would expect this to result in relatively low capture percentages for the Robust policy. We consider safety stock parameters ranging from $\gamma=2.5$, a relaxed setting, down to $\gamma=1$, a stress setting. For each setting of the safety stock parameter we consider three possible settings for the uncertainty set parameter: $\delta \in\{\gamma-0.1, \gamma, \gamma+0.1\}$. This is by way testing a conjecture that the best choice of $\delta$ is $\delta=\gamma$.

Table 6 presents the results. As anticipated, all of the Robust capture percentages are relatively low (under $20 \%$ ) because of the high coefficient of variation and the choice of period length. Of more interest is the direction of change in capture percentages with respect to local changes in the uncertainty set parameter about the value of the safety stock parameter. Without exception, increasing the uncertainty set parameter, $\delta$, in the neighborhood of $\gamma$ leads to a decrease in the Robust capture percentage, $C^{R}$, but an increase in the Robust terminal capture percentage, $C_{T}^{R}$. It is outside the scope of this paper to recommend a setting for the uncertainty set parameter; it appears to be a management parameter with interesting tradeoffs.

\subsubsection{Eight Pareto Retailers, Two Pareto Periods}

In this set of experiments, we consider eight non-identical retailers with $\beta_{D}=$ 0.8 . The daily demand rates that result from this setting are shown in Table 7 . As motivated in the appendix, we refer to this case as having Pareto retailers 


\begin{tabular}{|c|c|c|c|c|c|c|}
\hline$\gamma$ & $\delta$ & $C^{R}$ & $\mathbf{C}_{T}^{R}$ & $\mathbf{F}_{T}^{B}$ & $\mathbf{F}_{T}^{A}$ & $\mathbf{F}_{T}^{R}$ \\
\hline 2.5 & 2.4 & $\begin{array}{l}12.4 \\
\pm 2.0\end{array}$ & $\begin{array}{l}85.5 \\
\pm 1.0\end{array}$ & $\begin{array}{l}99.53 \\
\pm 0.05\end{array}$ & $\begin{array}{l}95.71 \\
\pm 0.21\end{array}$ & $\begin{array}{r}98.97 \\
\pm 0.07\end{array}$ \\
\hline 2.5 & 2.5 & $\begin{array}{c}8.8 \\
\pm 2.1\end{array}$ & $\begin{array}{l}86.5 \\
\pm 1.0\end{array}$ & $"$ & $"$ & $\begin{array}{l}99.01 \\
\pm 0.07\end{array}$ \\
\hline 2.5 & 2.6 & $\begin{array}{c}5.0 \\
\pm 2.3\end{array}$ & $\begin{array}{l}87.3 \\
\pm 1.0\end{array}$ & $"$ & $"$ & $\begin{array}{c}99.04 \\
\pm 0.06\end{array}$ \\
\hline 2.0 & 1.9 & $\begin{array}{l}19.0 \\
\pm 1.7\end{array}$ & $\begin{array}{l}75.7 \\
\pm 1.2\end{array}$ & $\begin{array}{l}98.70 \\
\pm 0.07\end{array}$ & $\begin{array}{c}92.92 \\
\pm 0.26\end{array}$ & $\begin{array}{l}97.29 \\
\pm 0.13\end{array}$ \\
\hline 2.0 & 2.0 & $\begin{array}{l}16.5 \\
\pm 1.8\end{array}$ & $\begin{array}{l}77.3 \\
\pm 1.2\end{array}$ & $"$ & $"$ & $\begin{array}{c}97.38 \\
\pm 0.12\end{array}$ \\
\hline 2.0 & 2.1 & $\begin{array}{c}13.8 \\
\pm 1.9\end{array}$ & $\begin{array}{l}78.7 \\
\pm 1.2\end{array}$ & $"$ & $"$ & $\begin{array}{l}97.45 \\
\pm 0.12\end{array}$ \\
\hline 1.5 & 1.4 & $\begin{array}{l}18.0 \\
\pm 1.1\end{array}$ & $\begin{array}{c}60.8 \\
\pm 0.9\end{array}$ & $\begin{array}{l}96.85 \\
\pm 0.13\end{array}$ & $\begin{array}{l}88.79 \\
\pm 0.30\end{array}$ & $\begin{array}{c}93.69 \\
\pm 0.23\end{array}$ \\
\hline 1.5 & 1.5 & $\begin{array}{c}16.8 \\
\pm 1.2\end{array}$ & $\begin{array}{c}63.3 \\
\pm 1.0\end{array}$ & $"$ & $"$ & $\begin{array}{c}93.89 \\
\pm 0.22\end{array}$ \\
\hline 1.5 & 1.6 & $\begin{array}{l}15.3 \\
\pm 1.3\end{array}$ & $\begin{array}{l}65.7 \\
\pm 1.0\end{array}$ & $"$ & $"$ & $\begin{array}{l}94.08 \\
\pm 0.22\end{array}$ \\
\hline 1.0 & 0.9 & $\begin{array}{l}12.0 \\
\pm 0.6\end{array}$ & $\begin{array}{l}42.0 \\
\pm 0.6\end{array}$ & $\begin{array}{l}93.12 \\
\pm 0.25\end{array}$ & $\begin{array}{l}82.97 \\
\pm 0.33\end{array}$ & $\begin{array}{l}87.24 \\
\pm 0.33\end{array}$ \\
\hline 1.0 & 1.0 & $\begin{array}{l}11.7 \\
\pm 0.7\end{array}$ & $\begin{array}{l}45.5 \\
\pm 0.6\end{array}$ & $"$ & $"$ & $\begin{array}{l}87.58 \\
\pm 0.33\end{array}$ \\
\hline 1.0 & 1.1 & $\begin{array}{c}11.1 \\
\pm 0.7\end{array}$ & $\begin{array}{l}48.7 \\
\pm 0.7\end{array}$ & $"$ & $"$ & $\begin{array}{c}87.91 \\
\pm 0.33\end{array}$ \\
\hline
\end{tabular}

Table 6: Simulation results for identical retailers, two Pareto-length periods, a high coefficient of variation, and various combinations of safety stock factor and uncertainty set parameter, showing robust capture percentage, $C^{R}$, robust terminal capture percentage, $C_{T}^{R}$, and terminal fill rates, $F_{T}^{B}, F_{T}^{A}$, and $F_{T}^{R}$. 


\begin{tabular}{|c|c|c|c|c|c|c|c|c|}
\hline & $\mathbf{1}$ & $\mathbf{2}$ & $\mathbf{3}$ & $\mathbf{4}$ & $\mathbf{5}$ & $\mathbf{6}$ & $\mathbf{7}$ & $\mathbf{8}$ \\
\hline Mean & 22.08 & 9.91 & 4.45 & 2.00 & 0.90 & 0.40 & 0.18 & 0.08 \\
\hline C.V. & 0.18 & 0.27 & 0.41 & 0.60 & 0.90 & 1.35 & 2.01 & 3.00 \\
\hline
\end{tabular}

Table 7: Daily demand mean and coefficient of variation by retailer when $N=8$, $\mu=5, \beta_{D}=0.8$, and $\psi=3$.

\begin{tabular}{|l|c|c|c|c|c|}
\hline$\gamma$ & $C^{R}$ & $\mathbf{C}_{T}^{R}$ & $\mathbf{F}_{T}^{B}$ & $C^{M}$ & $C_{T}^{M}$ \\
\hline \multirow{2}{*}{2} & -42.5 & 98.6 & 99.84 & -351.8 & 99.5 \\
& \pm 3.7 & \pm 0.2 & \pm 0.01 & \pm 9.17 & \pm 0.1 \\
\hline \multirow{2}{*}{1.5} & -63.5 & 98.8 & 99.65 & -232.9 & 99.0 \\
& \pm 3.2 & \pm 0.2 & \pm 0.01 & \pm 4.8 & \pm 0.1 \\
\hline
\end{tabular}

Table 8: Simulation results for eight Pareto retailers, two Pareto periods, high $\mathrm{COV}$, and two safety stock factors, $\gamma$, showing robust capture percentage, $C^{R}$, robust terminal capture percentage, $C_{T}^{R}$, and rebalance terminal fill rate, $F_{T}^{B}$, Ship Mean capture percentage, $C^{M}$, and Ship Mean terminal capture percentage, $C_{T}^{M}$.

since they follow an 80-20 rule with respect to demand rates. We consider a high coefficient of variation, $\psi=3$, but this is only for the smallest retailer. As the table shows, our test case generation approach assumes the larger retailers will have lower coefficients of variation. We also consider two Pareto periods, with $\beta_{L}=0.8$. Because of the computational effort involved in the eight retailer scenario, we limit consideration to only two settings of the safety stock parameter: $\gamma=1.5$ and 2 .

Table 8 shows the results. In this case, the Robust capture percentages are negative: the Robust policy performs worse than the Ship All policy with respect to time-weighted backorders. On the other hand, it performs quite well with respect to terminal backorders (greater than $98.5 \%$ in both cases). This suggests that it is conservative in reserving stock. The Ship Mean is an extreme example of a conservative policy so we include its performance in the table as well. The Ship Mean terminal capture percentages are marginally better than the Robust policy but the Ship Mean capture percentages (i.e. for timeweighted backorders) are a factor of 10 worse. The Robust policy is not nearly as conservative as the Ship Mean policy.

\subsubsection{Four Retailers with Varying Demand Shape Parameters}

In order to further explore the phenomenon discovered in the previous experiment, we consider a range of demand shape parameters varying from $\beta_{D}=0.2$ (identical retailers) to $\beta_{D}=0.8$ (Pareto retailers), but limited to four retailers. As in the previous section, we set the coefficient of variation of the smallest retailer, $\psi=3$. Table 9 displays the results. The ideal fill rate, $F_{T}^{B}$, from the Rebalancing policy reveals that the allocation problem becomes progressively easier as demand among the retailers becomes more unbalanced. This can be 


\begin{tabular}{|c|c|c|c|c|c|}
\hline$\beta_{D}$ & $C^{R}$ & $\mathbf{C}_{T}^{R}$ & $\mathbf{F}_{T}^{B}$ & $\mathbf{F}_{T}^{A}$ & $\mathbf{F}_{T}^{R}$ \\
\hline \multirow{2}{*}{0.2} & 16.53 & 77.26 & 98.70 & 92.92 & 97.34 \\
& \pm 1.77 & \pm 1.23 & \pm 0.07 & \pm 0.26 & \pm 0.12 \\
\hline \multirow{2}{*}{0.35} & 4.34 & 82.69 & 98.93 & 94.15 & 98.10 \\
& \pm 2.12 & \pm 1.16 & \pm 0.07 & \pm 0.22 & \pm 0.09 \\
\hline \multirow{2}{*}{0.5} & -38.95 & 90.30 & 99.30 & 96.27 & 99.01 \\
& \pm 3.63 & \pm 1.02 & \pm 0.05 & \pm 0.16 & \pm 0.06 \\
\hline \multirow{2}{*}{0.65} & -149.04 & 95.92 & 99.63 & 98.16 & 99.57 \\
& \pm 8.70 & \pm 0.86 & \pm 0.03 & \pm 0.09 & \pm 0.03 \\
\hline \multirow{2}{*}{0.8} & -122.62 & 95.33 & 99.87 & 99.44 & 99.85 \\
& \pm 9.25 & \pm 0.94 & \pm 0.01 & \pm 0.03 & \pm 0.01 \\
\hline
\end{tabular}

Table 9: Simulation results for Pareto retailers, Pareto period lengths, and varying shape parameters, $\beta_{D}$, showing robust capture percentage, $C^{R}$, robust terminal capture percentage, $C_{T}^{R}$, and terminal fill rates, $F_{T}^{B}, F_{T}^{A}$, and $F_{T}^{R}$.

explained in part because the coefficients of variation of demand for the larger retailers decrease. In other words, unbalancing the retailers is, in itself, a form of risk pooling because concentrating demand in a smaller number of retailers reduces the safety stock requirements. Furthermore, recall that system service levels can be increased by supplying retailers more frequently. Consequently, when demand rates are very low and coefficients of variation are very high, as for the low demand rate retailers in these examples, we recommend a policy of differentiation among retailers. Low demand rate retailers should be resupplied more frequently than high demand rate retailers.

\subsubsection{Computational Time}

Finally, we consider the computational time required to determine the Robust policy in a rolling horizon. For each test case and each demand sample we capture the elapsed time to compute the policy. We then compute the average elapsed time over all demand samples for each test case. Table 10 computes the mean and standard deviation of these test case averages across test cases with similar dimensions. As can be seen, there is a dramatic increase in computation time when the number of retailers is doubled. For eight retailers, the policy requires approximately one minute of computation time. Computing the policy for 10,000 samples requires approximately 180 hours on a single processor. For this reason, we have focused the studies on the cases with four retailers.

\subsubsection{Conclusions}

In this paper we have re-visited a long-standing multi-echelon inventory allocation problem and considered it afresh from a robust optimization perspective. We formulated the problem as a one warehouse, $N$-retailer, multi-period, stock allocation problem in which holding costs are identical at each location and no stock is received from outside suppliers for the duration of the planning horizon. 


\begin{tabular}{|l|c|l|l}
\hline \multicolumn{1}{|c|}{ Scenario } & $N=4$ & $N=4$ & $N=8$ \\
Dimensions & $T=2$ & $T=3$ & $T=2$ \\
\hline Mean & 1.66 & 8.13 & 64.57 \\
\hline Std.Dev. & 0.42 & 1.20 & 5.04 \\
\hline
\end{tabular}

Table 10: Computation time (in seconds) to compute the robust policy in a rolling horizon fashion for a single demand sample, averaged over samples and summarized by test case dimension.

Stock may be transferred from the central warehouse to the retailers instantaneously and without cost at the beginning of each period whenever the central warehouse still has stock on hand. No other stock transfers are allowed. Under this set-up, the only motive for holding inventory at the central warehouse for allocation in future periods is the so-called risk-pooling motive. The dynamic programming formulation of this problem requires a state space too large for practical computation. Various approximation methods have been proposed for variants of this problem. In this paper, beginning with a single period allocation problem, we have shown how to formulate both the objective function and the constraints needed to apply robust optimization to this problem. In particular, the objective of minimizing expected backorders is replaced by minimizing maximum backorders; the uncertainty set is expanded to include limits on partial sums of demand across retailers; and, the decision variables are partitioned into target inventory variables, which are set before demands are observed, and shipment variables, which are consequences of the inventory targets and the observed demands. The target inventory variables are optimized against the worst case observed demands. We have shown how to represent the uncertainty set compactly so that it grows by no more than the square of the number of retailers. The problem can be solved using Benders' decomposition employing a linear program as the master problem and a mixed-integer linear program as the subproblem. In the special case of no initial retailer inventories, two periods, and identical retailers, a relaxed form of the problem admits a closed form solution. A surprising result of this special case is that the optimal stock to hold in reserve at the central warehouse for allocation to retailers in the second period is invariant to the initial stock at the warehouse. Furthermore, this stock is proportional to the square root of the number of retailers. Risk pooling is captured in the formula through the interesting result that the worst case number of retailers to participate in the period 2 allocation is exactly $N / 2$ in the relaxed problem.

Summarizing the experimental results of the paper, we see both confirmation of the value of the robust optimization approach as well as managerial insights into the design and operation of multi-echelon inventory systems. In contrast to previous studies, we find that the Ship All policy, which ignores the riskpooling phenomenon, works well in systems with highly unbalanced demand rates across retailers. This is because the act of concentrating demand in a small number of retailers is, in itself, a form of risk pooling: the coefficient of 
variation of demand at the larger retailers can be expected to be much smaller than at the smaller retailers. The Robust policy performs worse than the Ship All policy in highly unbalanced systems: it is too conservative in reserving stock centrally. However, we also observed that system service levels can be increased by supplying retailers more frequently. Consequently, when demand rates are very low and coefficients of variation are very high, we recommend a policy of differentiation among retailers. Low demand rate retailers should be resupplied more frequently than high demand rate retailers. In all other situations considered, with identical demand rates across retailers, the Robust policy outperforms the Ship All policy both in terms of time-weighted backorders and terminal fill rate. The degree to which the Robust policy captures the riskpooling potential benefit is affected by the coefficient of variation of demand, the number of periods, the relative period lengths, the initial stock level, and the uncertainty bound.

Computation times increase in the number of retailers considered, but are small in comparison with dynamic programming approaches.

Future research on this topic should include comparison of the quality of robust solutions with those of approximate dynamic programming, scaling the algorithm and testing with large numbers of retailers, developing guidelines or heuristics for setting the uncertainty bound parameter, and extending the model to include procurement as well as allocation.

\section{References}

[Axsäter et al., 2002] Sven Axsäter, Johan Marklund, and Edward A. Silver. Heuristic methods for centralized control of one-warehouse, n-retailer inventory systems. Manufacturing $\mathcal{E}$ Service Operations Management, 4(1):75-97, 2002.

[Axsäter, 2003] Sven Axsäter. Supply chain operations: Serial and distribution systems. In S. C. Graves and A. G. de Kok, editors, Handbooks in OR and MS, Supply Chain Management: Design, Coordination and Operation, volume 11, chapter 10, pages 525-559. Elsevier Science Publishers B. V., Amsterdam, North Holland, 2003.

[Ben-Tal and Nemirovski, 2001] Aharon Ben-Tal and Arkadi Nemirovski. Lectures on Modern Convex Optimization: Analysis, Algorithms, and Engineering Applications. MOS-SIAM Series on Optimization. Society for Industrial and Applied Mathematics, Philadelphia, 2001.

[Bertsimas and Thiele, 2006] Dimitris Bertsimas and Aurélie Thiele. A robust optimization approach to inventory theory. Operations Research, 54(1):150168, January-February 2006.

[Bredström et al., 2013] D. Bredström, P. Flisberg, and M. Rönnqvist. A new method for robustness in rolling horizon planning. International Journal of Production Economics, 143(1):41 - 52, 2013. 
[Clark and Scarf, 1960] Andrew J. Clark and Herbert Scarf. Optimal policies for a multi-echelon inventory problem. Management Science, 6(4):475-490, 1960.

[Doğru et al., 2009] Mustafa K. Doğru, A.G. de Kok, and G.J. van Houtum. A numerical study on the effect of the balance assumption in one-warehouse multi-retailer inventory systems. Flexible Services and Manufacturing Journal, 21(3-4):114-147, 2009.

[Eppen and Schrage, 1981] Gary Eppen and Linus Schrage. Centralized ordering policies in a multi-warehouse system with lead times and random demand. In L.B. Schwarz, editor, Multi-Level Production/Inventory Systems: Theory and Practice, volume 16 of TIMS Studies in the Management Sciences, pages 51-67. North-Holland Publishing Company, 1981.

[Federgruen and Zipkin, 1984a] Awi Federgruen and Paul Zipkin. Allocation policies and cost approximations for multilocation inventory systems. Naval Research Logistics, 31(1):97-129, March 1984.

[Federgruen and Zipkin, 1984b] Awi Federgruen and Paul Zipkin. Approximations of dynamic, multilocation production and inventory problems. Management Science, 30(1):69-84, January 1984.

[Gallego et al., 2007] Guillermo Gallego, Özalp Özer, and Paul H. Zipkin. Bounds, heuristics, and approximations for distribution systems. Operations Research, 55(3):503-517, 2007.

[Jackson and Muckstadt, 1989] Peter L. Jackson and John A. Muckstadt. Risk pooling in a two-period, two-echelon inventory stocking and allocation problem. Naval Research Logistics, 36:1-26, 1989.

[Jönsson and Silver, 1987] H. Jönsson and E. A. Silver. Analysis of a twoechelon inventory control system with complete redistribution. Management Science, 33(2):215-227, 1987.

[Kunnumkal and Topaloglu, 2008] Sumit Kunnumkal and Huseyin Topaloglu. A duality-based relaxation and decomposition approach for inventory distribution systems. Naval Research Logistics, 55(7):612-631, October 2008.

[Kunnumkal and Topaloglu, 2011] Sumit Kunnumkal and Huseyin Topaloglu. Linear programming based decomposition methods for inventory distribution systems. European Journal of Operational Research, 211(2):282-297, June 2011.

[Zakeri et al., 2014] G. Zakeri, D. Craigie, A. Philpott, and M. Todd. Optimization of demand response through peak shaving. Operations Research Letters, 42(1):97-101, January 2014.

[Zipkin, 1984] Paul Zipkin. On the imbalance of inventories in multi-echelon systems. Mathematics of Operations Research, 9(3):402-423, 1984. 


\section{Appendices}

\subsection{Experimental Design}

In this section, we propose a model for generating test cases based on an economical set of generating parameters. Let $\mu_{i}$ denote the expected daily demand at retailer $i, i \in \mathcal{N}$. Let $N=|\mathcal{N}|$. Let $\bar{\mu}$ denote the average daily demand across retailers: $\bar{\mu}=N^{-1} \sum_{i=1}^{N} \mu_{i}$. We assume the distribution of demand across retailers, in expected value, follows a Pareto law:

$$
\mu_{i}=\alpha_{D}^{i-1} \mu_{1}
$$

for some $\alpha_{D} \leq 1$, for $i=1,2, \ldots, N$. When $\alpha_{D}=1$, the retailers are identical and $\mu_{1}=\bar{\mu}$. When $\alpha_{D}<1$, we have the relationships

$$
\sum_{i=1}^{N} \mu_{i}=\mu_{1} \frac{1-\alpha_{D}^{N}}{1-\alpha_{D}}
$$

and

$$
\mu_{1}=\frac{N \bar{\mu}\left(1-\alpha_{D}\right)}{1-\alpha_{D}^{N}}
$$

so the parameters $\bar{\mu}$ and $\alpha_{D}$ are sufficient to generate the complete vector of daily demands, $\left(\mu_{1}, \ldots, \mu_{N}\right)$. We seek to create demand distributions for which some prespecified fraction, $\beta_{D}$, of total demand is concentrated in the largest $20 \%$ of retailers. That is, we look to create distributions satisfying the relation

$$
\frac{\sum_{i=1}^{m} \mu_{i}}{\sum_{i=1}^{N} \mu_{i}}=\beta,
$$

where $m$ is approximately $20 \%$ of $N$. This reduces to finding a value of $\alpha_{D}$, which we call $\alpha_{D}(\beta)$, which satisfies

$$
\lim _{\alpha \rightarrow \alpha_{D}} \frac{1-\alpha^{\lceil 0.2 N\rceil}}{1-\alpha^{N}}=\beta_{D} .
$$

This equation does not have an analytical solution but can be solved easily by a search procedure. Observe that $\beta_{D}=0.8$ will approximate the common $80-20$ Pareto distribution and $\beta_{D}=0.2$ will yield $\alpha_{D}\left(\beta_{D}\right)=1$ (the limit of the left hand side, as $\alpha_{D}$ approaches 1, by L'Hôpital's Rule, is 0.2). As a result, the two parameters $\bar{\mu}$ and $\beta_{D}$ are sufficient to generate the complete vector of daily demand means. We refer to $\beta_{D}$ as the Pareto demand shaping parameter.

Let $\psi$ denote the coefficient of variation of daily demand of the smallest retailer. The standard deviation of daily demand of the smallest retailer is therefore $\psi \mu_{N}$. The variance of demand of the smallest retailer is $\left(\psi \mu_{N}\right)^{2}$. As-

sume the variance of daily demand of retailer $i$ is given by $\frac{\mu_{i}}{\mu_{N}}\left(\psi \mu_{N}\right)^{2}$. For example, if a retailer has twice the rate of daily demand of retailer $N$, that is, $\mu_{i}=2 \mu_{N}$, then its demand behaves like the sum of two independent demand 
streams each with the standard deviation of the smallest retailer. Let $\sigma_{i}$ denote the standard deviation of daily demand for retailer $i$. According to the previous logic, we have

$$
\sigma_{i}=\psi \sqrt{\mu_{i} \mu_{N}},
$$

$i=1, \ldots, N$. Consequently, given the vector of demand rates, the parameter $\psi$ is sufficient to generate reasonable choices for daily demand standard deviations.

Let $T$ denote the number of allocation periods in the problem. Let $l_{t}$ denote the length (number of days) of allocation period $t$. We assume that the periods are non-increasing in length: $l_{1} \geq l_{2} \geq \ldots \geq l_{T}$. In parallel fashion to the way we generate the mean daily demand parameters, we assume we are given the average number of days per period, $\bar{l}$, and a Pareto period length shaping parameter, $\beta_{L}$. From these, we determine a value of $\alpha_{L}$, called $\alpha_{L}\left(\beta_{L}\right)$, which solves

$$
\lim _{\alpha \rightarrow \alpha_{L}} \frac{1-\alpha^{\lceil 0.2 T\rceil}}{1-\alpha^{T}}=\beta_{L}
$$

and the length of the first period, $l_{1}$, using

$$
l_{1}=\frac{T \bar{l}\left(1-\alpha_{L}\left(\beta_{L}\right)\right)}{1-\alpha_{L}\left(\beta_{L}\right)^{T}} .
$$

The remaining period lengths are given by $l_{t}=\alpha_{L}\left(\beta_{L}\right)^{t-1} l_{1}, t=2,3, \ldots, T$. Setting $\beta_{L}=0.2$ will result in period lengths being identical. We do not require period lengths to be integer because the period length is used only to scale demand rates.

Combining the daily demand rates with the period lengths permits us to generate the period demand rates required by the optimization model:

$$
\mu_{i t}=l_{t} \mu_{i}, i=1,2, \ldots, N ; t=1,2, \ldots, T \text {. }
$$

Similarly, assuming daily demands are independent and identically distributed over time, the standard deviations of period demands are given by

$$
\sigma_{i t}=\sqrt{l_{t}} \sigma_{i}, i=1,2, \ldots, N ; t=1,2, \ldots, T \text {. }
$$

In summary, using this test case model, the demand parameters for the optimization model can be generated from the following seven test parameters: $n, \bar{\mu}, \beta_{D}, \psi, T, \bar{l}$, and $\beta_{L}$.

Considering the initial conditions for the optimization model, we assume that initial inventories at all retailers are zero in all test cases: $\mathrm{v}_{i}=0, i \in \mathcal{N}$. This is equivalent to assuming that the delivery of stock which initiates the allocation cycle is sufficient to raise all retailers to their ideal target inventories and that no retailer has more than its ideal target level. The ideals are computed relative to the total amount of inventory in the system. So, for simplicity, we assume all inventory in the system is initially concentrated at the central warehouse. We express the initial system reserve stock, $v_{0}$, in terms of a parameter $\gamma$, the 
safety stock factor under the assumption that all demand is concentrated in a single location and time period:

$$
v_{0}=T \bar{l} N \bar{\mu}+\gamma \sqrt{\sum_{t=1}^{T} l_{t} \sum_{i \in \mathcal{N}} \sigma_{i}^{2}} .
$$

That is, $\gamma$ is the number of standard deviations of total system demand to hold as safety stock. Assuming that normal operation of the system would result in at least two standard deviations of total system demand, we assume that $\gamma \leq 1$ would represent a system under stress and $\gamma \geq 3$ would represent a system flush with stock.

Substituting sequentially for $\sigma_{i}, \mu_{N}$ and $\mu_{1}$ from (34), (32), and (33), respectively, yields

$$
\begin{aligned}
v_{0} & =T \bar{l} N \bar{\mu}+\gamma \sqrt{T \bar{l} \psi^{2} \mu_{N} \sum_{i \in \mathcal{N}} \mu_{i}} \\
& =T \bar{l} N \bar{\mu}+\gamma \psi \sqrt{T \bar{l} \mu_{N} N \bar{\mu}} \\
& =T \bar{l} N \bar{\mu}+\gamma \psi \sqrt{T \bar{l} \alpha^{N-1} \mu_{1} N \bar{\mu}} \\
& =T \bar{l} N \bar{\mu}+\gamma \psi N \bar{\mu} \lim _{\alpha \rightarrow \alpha_{D}\left(\beta_{D}\right)} \sqrt{T \bar{l} \alpha^{N-1} \frac{(1-\alpha)}{1-\alpha^{N}}} .
\end{aligned}
$$

For the special case of identical retailers, $\beta_{D}=0.2$ and $\alpha_{D}\left(\beta_{D}\right)=1$, this reduces to

$$
v_{0}=T \bar{l} N \bar{\mu}+\gamma \psi \bar{\mu} \sqrt{T \bar{l} N} .
$$

The optimization algorithm can be tuned by choosing different values of $\delta$, the uncertainty set parameter. Two natural values to consider would be $\delta=2$ and $\delta=\gamma$. The optimization algorithm can be further tuned by choosing different values for the time period weights, $w_{t}, t=1,2, \ldots, T$. We consider the following time period weighting scheme: $w_{t}=t^{\theta}, t=1,2, \ldots, T$. Hence, $\theta=0$ generates equal weights, $\theta>0$ generates weights which increase over time, and $\theta<0$ generates weights which decrease over time. Finally, in the case of non-identical retailers, the algorithm can be tuned by choosing retailer-specific weights. Motivated by Corollary 4, we propose the following scheme:

$$
w_{i t}=\frac{w_{t}}{\sigma_{i t}^{\omega}}
$$

for every $i \in \mathcal{N}, t=1,2, \ldots, T$, where $\omega \in\{0,1\}$, a weighting scale exponent. Setting $\omega=0$ results in $w_{i t}=w_{t}$ for all $i \in \mathcal{N}$; i.e. it corresponds to no weight adjustment at the retailer level. Setting $\omega=1$, adjusts the weights as suggested by Corollary 4 .

Table 11 summarizes the parameters used to generate test cases. 


\begin{tabular}{ll}
\hline Parameter Name & Symbol \\
\hline Number of retailers & $N$ \\
\hline Number of periods & $T$ \\
\hline Mean daily demand & $\bar{\mu}$ \\
\hline Mean days per period & $\bar{l}$ \\
\hline Coefficient of variation & $\psi$ \\
\hline Pareto demand shape & $\beta_{D}$ \\
\hline Pareto period-length shape & $\beta_{L}$ \\
\hline Safety stock factor & $\gamma$ \\
\hline
\end{tabular}

Table 11: Parameters and symbols for generating test cases.

\subsection{Standard Proofs}

Many of the results of the paper are established using standard proof approaches and are not particularly instructive. We collect such proofs in this appendix to allow a more focused presentation in the main body of the paper.

\subsubsection{Proposition 1}

Proof. Let $F_{i}\left(x_{i} ; v_{i}\right)=E\left[\left(\widetilde{d}_{i}-v_{i}-x_{i}\right)^{+}\right], i \in \mathcal{N}$. Then we wish to solve

$$
\min _{\substack{x_{i} \geq 0, i \in \mathcal{N} \\ \sum_{i \in \mathcal{N}} x_{i} \leq v_{0}}} \sum_{i \in \mathcal{N}} F_{i}\left(x_{i} ; v_{i}\right) .
$$

The Karush-Kuhn-Tucker conditions for optimality imply $\frac{d F_{i}}{d x_{i}}+\theta \geq 0$, for all $i \in \mathcal{N}$, with equality holding if $x_{i}>0$, where $\theta$ is the Lagrange multiplier corresponding to the constraint $\sum_{i \in \widetilde{\mathcal{N}}} x_{i} \leq v_{0}$ and $\theta$ is non-negative. Expanding $F_{i}$, we have

$$
\begin{aligned}
F_{i}\left(x_{i} ; v_{i}\right) & =\int_{v_{i}+x_{i}}^{\infty}\left(d_{i}-v_{i}-x_{i}\right) f_{i}\left(d_{i}\right) d d_{i} \\
& =\int_{\frac{v_{i}+x_{i}-\mu_{i}}{\sigma_{i}}}^{\infty}\left(\mu_{i}+\sigma_{i} \varepsilon-v_{i}-x_{i}\right) g(\varepsilon) d \varepsilon,
\end{aligned}
$$

where $f_{i}(\cdot)$ is the density function for the random variable $\widetilde{d}_{i}$ and $g(\cdot)$ is the common density function for the random variable $\widetilde{\varepsilon}$. The derivative is given by

$$
\begin{aligned}
\frac{d F_{i}}{d x_{i}} & =-\int_{\frac{v_{i}+x_{i}-\mu_{i}}{\sigma_{i}}}^{\infty} g(\varepsilon) d \varepsilon \\
& =-\bar{G}\left(\frac{v_{i}+x_{i}-\mu_{i}}{\sigma_{i}}\right)
\end{aligned}
$$

Let $y_{i}=v_{i}+x_{i}$. Then the optimality condition states that

$$
\bar{G}\left(\frac{y_{i}-\mu_{i}}{\sigma_{i}}\right) \leq \theta
$$


for all $i \in \mathcal{N}$, with equality holding if $y_{i}>v_{i}$. By assumption, $\bar{G}(\varepsilon)>0$ for all $\varepsilon$, so we must have $\theta>0$ in the optimal solution and, hence the constraint $\sum_{i \in \mathcal{N}} x_{i} \leq v_{0}$ must be satisfied with equality. Let $\widetilde{\mathcal{N}}$ be the set of retailers for which $y_{i}>v_{i}$, that is, given $y, \widetilde{\mathcal{N}}$ is the set of retailers which receive a positive allocation. Thus, $\bar{G}\left(\frac{y_{i}-\mu_{i}}{\sigma_{i}}\right)$ are equal for all $i \in \widetilde{\mathcal{N}}$ and $\theta$ provides the common fractile value. Hence, the optimal value $y_{i}^{*}$ satisfies $y_{i}^{*}=\mu_{i}+\bar{G}^{-1}(\theta) \sigma_{i}$ for all $i \in \widetilde{\mathcal{N}}$. Noting $x_{i}^{*}=y_{i}^{*}-v_{i}$ and the definition of $\widetilde{\mathcal{N}}$, the result follows quickly.

\subsubsection{Proposition 2.}

Proof. Clearly, the worst case demand satisfies $\varepsilon_{i}^{*}=\delta$, for all $i \in \mathcal{N}$. Furthermore, if $B^{*}>0$ then $S\left(y^{*}\right)=v_{0}$. For $y_{i}^{*}$ to be feasible, we must have

$$
y_{i}^{*} \geq \mu_{i}+\sigma_{i} \delta-w_{i}^{-1} B^{*} .
$$

Suppose, by way of contradiction, that $y_{i}^{*}>\mu_{i}+\sigma_{i} \delta-w_{i}^{-1} B^{*}$ for some $i$. If $y_{i}^{*} \leq v_{i}$, then $x_{i}^{*}=0$ in the solution to $S(y)$ and $y_{i}^{*}$ can be decreased without changing either $S(y)$ or the value of $B^{*}$. On the other hand, if $y_{i}^{*}>v_{i}$ then the corresponding shipment $x_{i}^{*}=y_{i}^{*}-v_{i}$ is positive. If $B^{*}>0$, then, by decreasing $y_{i}^{*}$, it would be possible to increase the shipments to all other retailers, $j$, for which $y_{j}^{*}=\mu_{j}+\sigma_{j} \delta-w_{j}^{-1} B^{*}$. It follows that it would thereby be possible to decrease $B^{*}$ thus contradicting its optimality. Alternatively, if $B^{*}=0$, then, by decreasing $y_{i}^{*}, S(y)$ can be decreased without any change to $B^{*}$. Hence, it must be possible to find an optimal solution satisfying the stated condition.

\subsubsection{Corollary 4}

Proof. In this situation, $y_{i}(B)=\mu_{i}+\left(\delta-w^{-1} B\right) \sigma_{i}$. Comparing this with (1), we let

$$
\theta=\bar{G}\left(\delta-w^{-1} B^{*}\right) .
$$

Then $y_{i}(\theta)=y_{i}\left(B^{*}\right)$ and, by Corollary $3, \theta$ satisfies (2).

\subsubsection{Proposition 8}

Proof. Both the linear program and the expression for $S(y, \varepsilon)$ are separable by retailer. Let $S_{i}\left(y_{i}, \varepsilon_{i}\right)$ denote the problem restricted to retailer $i$. The solution (4) is feasible for the linear program and so $S_{i}\left(y_{i}, \varepsilon_{i}\right)$ is an upper bound on the linear programming solution restricted to retailer $i$. Let $x_{i}^{*}$ denote an optimal solution to the linear program. Let $t$ be the first period for which $x_{i t}^{*} \neq x_{i t}(\varepsilon)$. Since $x_{i t}(\varepsilon)$ is minimal, we must have $x_{i t}^{*}>x_{i t}(\varepsilon)$. Define a new solution $x_{i}^{\prime}$ reducing the $t^{t h}$ component of $x_{i}^{*}$ by $x_{i t}^{*}-x_{i t}(\varepsilon)$ and increasing the $t+1^{s t}$ component of $x_{i}^{*}$ by the same quantity. It is easily verified that this solution, $x_{i}^{\prime}$, is also optimal but now differs from $x_{i}(\varepsilon)$ starting in the $t+1^{\text {st }}$ component. Continuing in this manner, we find that $x_{i}(\varepsilon)$ is optimal for the linear program and so $S_{i}\left(y_{i}, \varepsilon_{i}\right)$ must equal the optimal objective value of the linear program restricted to retailer $i$. 


\subsubsection{Proposition 10}

Proof. If $t_{i}^{*}(\varepsilon)=0$, then no objective coefficient in (6) is positive for retailer $i$. In this case the optimal solution will be to set $\pi_{i t}^{*}(\varepsilon)=0$ for all $t=1,2, \ldots, T$. If $t_{i}^{*}(\varepsilon)>0$, then a marginal analysis argument can be used to show that, barring ties, any solution in which more than one $\pi_{i t}>0$ for any $i$ can be improved by increasing the $\pi_{i t}$ for the period $t$ with the largest objective coefficient, $t_{i}^{*}(\varepsilon)$, and decreasing the others. In the case of ties, the solution is unchanged by increasing one of the tied variables and decreasing the others. Hence, for each $i \in \mathcal{N}$, an optimal solution consists of setting one $\pi_{i t}=1$ and the rest equal to zero.

\subsubsection{Proposition 12}

Proof. By (5)

$$
\sum_{t^{\prime}=1}^{t} x_{i t^{\prime}}(\varepsilon) \geq y_{i t}-v_{i}+\sum_{t^{\prime}=1}^{t-1}\left(\mu_{i t^{\prime}}+\sigma_{i t^{\prime}} \varepsilon_{i t^{\prime}}\right)
$$

with equality holding if $x_{i t}(\varepsilon)>0$. Furthermore, $\sum_{t^{\prime}=1}^{t} x_{i t^{\prime}}(\varepsilon)$ is non-decreasing in $t$. Suppose $\sum_{t=1}^{T} x_{i t}(\varepsilon)>0$. Let $\tau=\tau_{i}(\varepsilon)$ denote the last period in which $x_{i t}(\varepsilon)>0$. It follows that

$$
\sum_{t^{\prime}=1}^{\tau} x_{i t^{\prime}}(\varepsilon)=S_{i}(y, \varepsilon)=\max _{t=1,2, \ldots, T}\left(y_{i t}-v_{i}+\sum_{t^{\prime}=1}^{t-1}\left(\mu_{i t^{\prime}}+\sigma_{i t^{\prime}} \varepsilon_{i t^{\prime}}\right)\right)
$$

where $S_{i}(y, \varepsilon)$ is the required shipment for location $i$. Note that we have dropped the positive part function under the assumption that $x_{i t}(\varepsilon)>0$. It follows that for this value of $\tau$ we have

$$
y_{i \tau}-v_{i}+\sum_{t^{\prime}=1}^{\tau-1}\left(\mu_{i t^{\prime}}+\sigma_{i t^{\prime}} \varepsilon_{i t^{\prime}}\right)=\max _{t=1,2, \ldots, T}\left(y_{i t}-v_{i}+\sum_{t^{\prime}=1}^{t-1}\left(\mu_{i t^{\prime}}+\sigma_{i t^{\prime}} \varepsilon_{i t^{\prime}}\right)\right) .
$$

That is, $\tau$ is a candidate for $t_{i}^{*}(\varepsilon)$. In the case of ties, we defined $t_{i}^{*}(\varepsilon)$ to be the earliest period $t$ optimizing the right hand side. Consequently, $t_{i}^{*}(\varepsilon) \leq \tau$. Suppose, by way of contradiction, that $t_{i}^{*}(\varepsilon)<\tau$. In that case, we would have, by (5),

$$
\begin{aligned}
\sum_{t^{\prime}=1}^{t_{i}^{*}(\varepsilon)} x_{i t^{\prime}}(\varepsilon) & \geq y_{i t_{i}^{*}(\varepsilon)}-v_{i}+\sum_{t^{\prime}=1}^{t_{i}^{*}(\varepsilon)-1}\left(\mu_{i t^{\prime}}+\sigma_{i t^{\prime}} \varepsilon_{i t^{\prime}}\right) \\
& =\sum_{t^{\prime}=1}^{\tau} x_{i t^{\prime}}(\varepsilon)
\end{aligned}
$$

leading to the conclusion

$$
\sum_{t^{\prime}=t_{i}^{*}(\varepsilon)+1}^{\tau} x_{i t^{\prime}}(\varepsilon)=0
$$


which contradicts the assumption that $x_{i \tau}(\varepsilon)>0$. Consequently, $t_{i}^{*}(\varepsilon)=\tau$. On the other hand, if $\sum_{t=1}^{T} x_{i t}(\varepsilon)=0$, then $\tau_{i}(\varepsilon)=0$, by definition, and the result holds trivially.

\subsubsection{Proposition 15}

Proof. This is a straightforward consequence of the fact that $S(y)$ is nondecreasing in $y$. That is, if $\left(y^{0}, B^{*}\right)$ is any optimal solution to $(7)$ with $y_{i t}^{0}>$ $\bar{d}_{i t}(\delta)-w_{i t}^{-1} B_{t}^{*}$, then it can be shown that $y^{*}$ as defined by (8) satisfies $y^{*} \leq y^{0}$ and, hence, $S\left(y^{*}\right) \leq S\left(y^{0}\right) \leq v_{0}$. The combination $\left(y^{*}, B^{*}\right)$ is therefore feasible and yields the same objective value as the optimal solution $\left(y^{0}, B^{*}\right)$.

\subsubsection{Proposition 16}

Proof. Denote an optimal solution to the MILP using the superscript "*". The binary variables $u_{i t}^{*}$ act as selectors. For each $i \in \mathcal{N}$, there will be exactly one $u_{i t}^{*}=0$, and hence if $t>0$, then $S_{i}^{*}=y_{i t}-v_{i}+\sum_{t^{\prime}=1}^{t-1}\left(\mu_{i t^{\prime}}+\sigma_{i t^{\prime}} \varepsilon_{i t^{\prime}}^{*}\right)$ for that value of $t$. If $u_{i 0}=0$, then $S_{i}^{*}=0$. It follows that

$$
S_{i}^{*}=\max _{t=1,2, \ldots, T}\left(y_{i t}-v_{i}+\sum_{t^{\prime}=1}^{t-1}\left(\mu_{i t^{\prime}}+\sigma_{i t^{\prime}} \varepsilon_{i t^{\prime}}^{*}\right)\right)^{+}
$$

and that $S(y)=\sum_{i \in \mathcal{N}} S_{i}^{*}$.

\subsubsection{Proposition 18}

Proof. Initially, i.e. on iteration $k=0$, we set $S_{\pi^{0}}(\cdot) \equiv 0$. That is, we ignore the shipment constraint on the first iteration. Let $\left(y^{k}, B^{k}\right)$ denote the optimal solution to the master problem on iteration $k$. The sub-problem at the $k^{\text {th }}$ iteration is to solve

$$
\max _{\pi \in \Pi} S_{\pi}\left(y^{k}\right)
$$

The optimal solution to the $k^{t h}$ sub-problem is denoted by $\pi^{k}$. It follows that if $S_{\pi^{k}}\left(y^{k}\right) \leq v_{0}$, then $\left(y^{k}, B^{k}\right)$ is an optimal solution to the original problem. In this case, the algorithm is terminated. On the other hand, if $S_{\pi^{k}}\left(y^{k}\right)>$ $v_{0}$, then, clearly, $\pi^{k} \notin \Pi^{k}$. Consequently, we extract the coefficients for $S_{\pi^{k}}(y)$ using (10) and add the constraint $S_{\pi^{k}}(y) \leq v_{0}$ to the master problem. We replace $\Pi^{k}$ with $\Pi^{k+1}=\Pi^{k} \cup\left\{\pi^{k}\right\}$ and repeat the process. Since $\Pi$ is finite and a new element, $\pi^{k}$, is identified on each iteration which fails to find an optimal solution, the algorithm must terminate with an optimal solution in a finite number of iterations. 


\subsubsection{Proposition 19}

Proof. The proposed solution satisfies the following constraints of (27) with equality:

$$
\begin{aligned}
& \varepsilon_{[1] 1}=\delta \\
& \varepsilon_{[1] 1}+\varepsilon_{[2] 1}=\sqrt{2} \delta \\
& \varepsilon_{[1] 1}+\varepsilon_{[2] 1}+\cdots+\varepsilon_{\left[n_{2}\right] 1}=\sqrt{n_{2}} \delta .
\end{aligned}
$$

These correspond to subsets $I=\{[1]\},\{[1],[2]\}, \ldots,\left\{[1],[2], \ldots,\left[n_{2}\right]\right\}$ of $I_{2}$, respectively. Denote the dual variables for these constraints by $\eta_{\{[1]\}}, \eta_{\{[1],[2]\}}, \ldots, \eta_{\left\{[1],[2], \ldots,\left[n_{2}\right]\right\}}$, respectively. Set all other dual variables, $\eta_{I}, I \subseteq I_{2}$, to zero and solve the dual equations to find:

$$
\begin{aligned}
\eta_{\left\{[1],[2], \ldots,\left[n_{2}\right]\right\}=} & \sigma_{\left[n_{2}\right] 1} \\
\eta_{\left\{[1],[2], \ldots,\left[n_{2}-1\right]\right\}}= & \sigma_{\left[n_{2}-1\right] 1}-\sigma_{\left[n_{2}\right] 1} \\
& \vdots \\
\eta_{\{[1]\}}= & \sigma_{[1] 1}-\sigma_{[2] 1} .
\end{aligned}
$$

By construction, $\eta \geq 0$ and so $\eta$ is dual feasible. The primal objective is given by

$$
\begin{aligned}
\sum_{j=1}^{n_{2}} \sigma_{[j] 1} \varepsilon_{[j] 1} & =\sum_{j=1}^{n_{2}} \sigma_{[j] 1} \delta(\sqrt{j}-\sqrt{j-1}) \\
& =\sum_{j=1}^{n_{2}-1}\left(\sigma_{[j] 1}-\sigma_{[j+1] 1}\right) \sqrt{j} \delta+\sigma_{\left[n_{2}\right] 1} \sqrt{n_{2}} \delta .
\end{aligned}
$$

The dual objective is given by

$$
\begin{aligned}
\sum_{I \subseteq I_{2}} \eta_{I} \sqrt{|I|} \delta & =\sum_{n=1}^{n_{2}} \sum_{\substack{I \subseteq I_{2} \\
|I|=n}} \eta_{I} \sqrt{|I|} \delta \\
& =\sum_{n=1}^{n_{2}} \eta_{\{[1],[2], \ldots,[n]\}} \sqrt{n} \delta \\
& =\sum_{n=1}^{n_{2}-1}\left(\sigma_{[n] 1}-\sigma_{[n+1] 1}\right) \sqrt{n} \delta+\sigma_{\left[n_{2}\right] 1} \sqrt{n_{2}} \delta
\end{aligned}
$$

The dual objective value is therefore equal to the primal objective value. By duality, we have found an optimal extreme point. 


\subsubsection{Corollary 21}

Proof. By (5),

$$
\sum_{t^{\prime}=1}^{2} x_{i t^{\prime}}(\varepsilon) \geq y_{i 2}+\mu_{i 1}+\sigma_{i 1} \varepsilon_{i 1}
$$

for each $i \in \mathcal{N}$. By Proposition (19), the optimal $\varepsilon$ is positive. Under the conditions of the corollary, therefore, for each $i \in \mathcal{N}$, we have

$$
\sum_{t^{\prime}=1}^{2} x_{i t^{\prime}}(\varepsilon)>0 .
$$

That is, the optimal $\varepsilon$ requires that the shipments to each retailer be strictly positive in at least one of the two periods. Consequently, by Corollary (13) at least one of $\pi_{i 1}$ or $\pi_{i 2}$ must equal 1 , for each $i \in \mathcal{N}$. 